\title{
Best Practices in Global Mental Health: An Exploratory Study of Recommendations for Psychologists
}

Kimberly Hook

Follow this and additional works at: https://ecommons.luc.edu/luc_diss

Part of the Counseling Psychology Commons

\section{Recommended Citation}

Hook, Kimberly, "Best Practices in Global Mental Health: An Exploratory Study of Recommendations for Psychologists" (2018). Dissertations. 2962.

https://ecommons.luc.edu/luc_diss/2962

This Dissertation is brought to you for free and open access by the Theses and Dissertations at Loyola eCommons. It has been accepted for inclusion in Dissertations by an authorized administrator of Loyola eCommons. For more information, please contact ecommons@luc.edu. (c) (i) $(9)$

This work is licensed under a Creative Commons Attribution-Noncommercial-No Derivative Works 3.0 License. Copyright $\odot 2018$ Kimberly Hook 
LOYOLA UNIVERSITY CHICAGO

\author{
BEST PRACTICES IN GLOBAL MENTAL HEALTH: \\ AN EXPLORATORY STUDY OF RECOMMENDATIONS \\ FOR PSYCHOLOGISTS
}

A DISSERTATION SUBMITTED TO

THE FACULTY OF THE GRADUATE SCHOOL

IN CANDIDACY FOR THE DEGREE OF

DOCTOR OF PHILOSOPHY

PROGRAM IN COUNSELING PSYCHOLOGY

BY

KIMBERLY M. HOOK

CHICAGO, IL

AUGUST 2018 
Copyright by Kimberly Hook, 2018 All rights reserved. 


\section{ACKNOWLEDGEMENTS}

I would like to express my gratitude to my committee members, Drs. Eunju Yoon and Michael Maher, for their support of this research. I would especially like to thank my committee chair, Dr. Elizabeth Vera, both for her encouragement throughout this project and throughout my time in the doctoral program; your mentorship has been the most significant aspect of my academic experience, and I appreciate the time and energy you have kindly offered to me.

I am grateful to the participants of this study. This dissertation could not have been completed without the openness and generosity of the nine professionals who gave of their time to discuss their experiences in global mental health. Each of your stories and experiences is inspiring to me, and I am confident that learning from your examples will directly my impact to engage meaningfully in our field.

Most significantly, I am deeply appreciative of my family, including my parents, Mike and Tricia Hook, and my sister, Kristina Hook. It is from all of you and from our experiences together as a family that my interest in the global community first developed, and it is through all of your examples that my commitment to underserved individuals grew. Throughout my entire life, you have freely given me your love, encouragement, and support. This project is a reflection of the ways that you have invested in my life, and I am fortunate to come from a family who has a solid foundation of loving each other well. 


\section{TABLE OF CONTENTS}

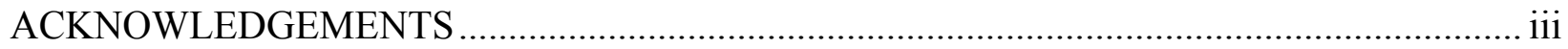

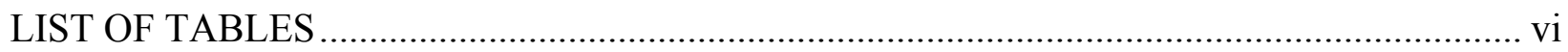

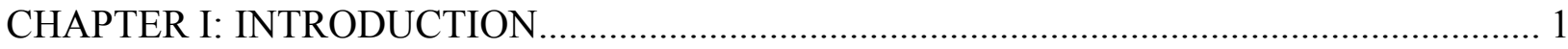

Growth of the Global Mental Health Field ............................................................................. 1

Mechanisms of Moving Towards a Global Mental Health Agenda....................................... 3

Challenges to Global Mental Health .................................................................................. 6

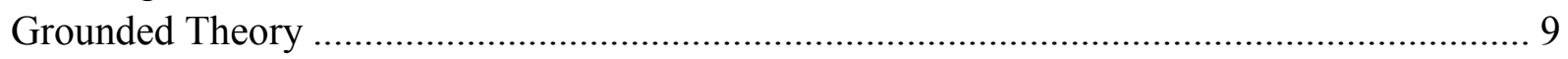

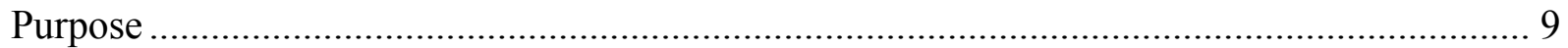

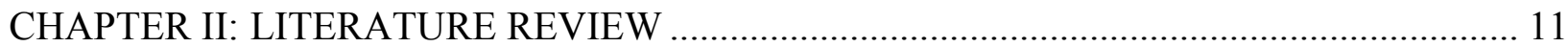

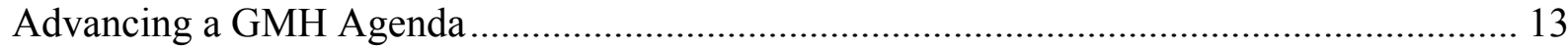

Critiques of the Field ................................................................................................ 14

Lack of Attention to Cultural and Contextual Factors.................................................... 14

Lack of Attention to Social Inequalities .................................................................... 16

Difficulty Extrapolating Western Interventions to Diverse Communities ........................ 17

Existing Recommendations for Practice .................................................................... 19

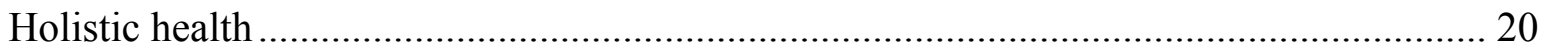

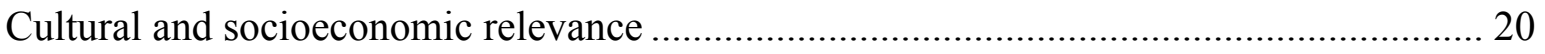

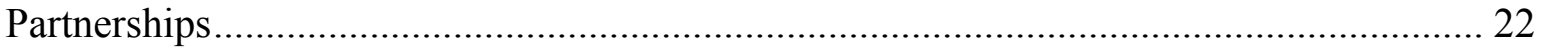

Collaborative action-based education and learning ...................................................... 23

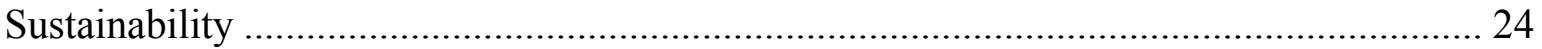

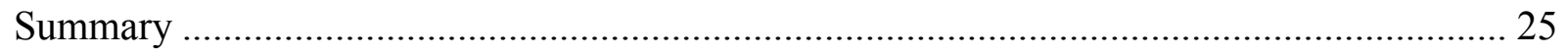

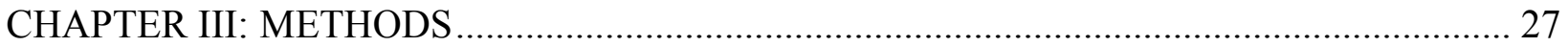

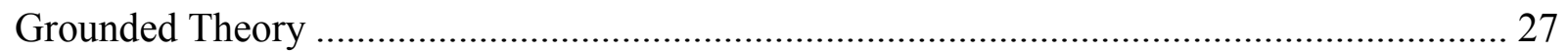

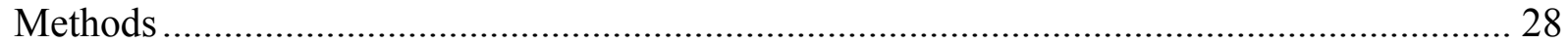

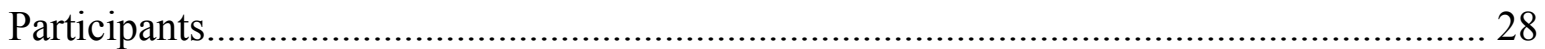

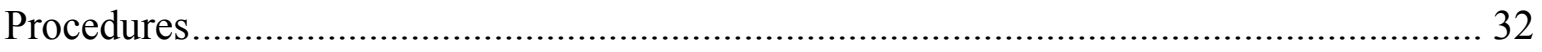

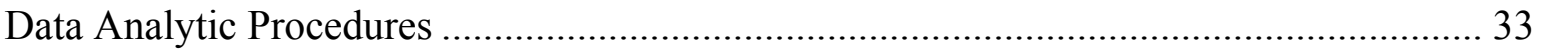

Researcher Background, Experiences and Biases ..................................................... 35

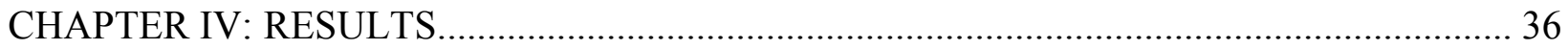

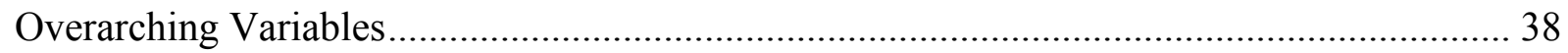

Consideration of Cultural/Contextual Variables........................................................... 38

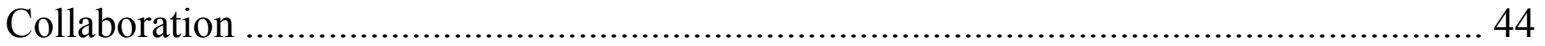

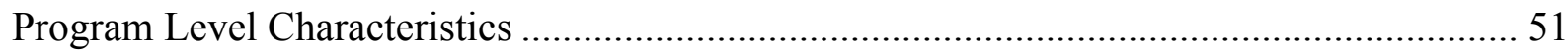

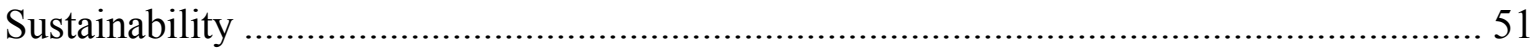

Evaluation and Research Methods....................................................................... 53

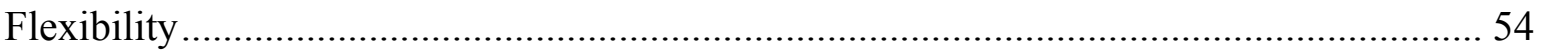




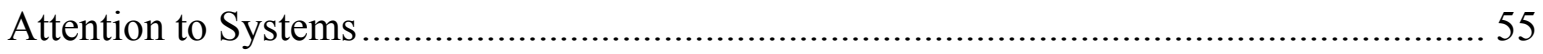

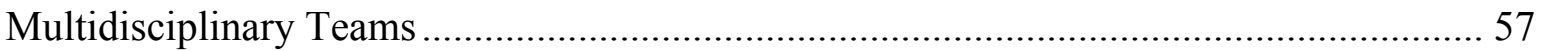

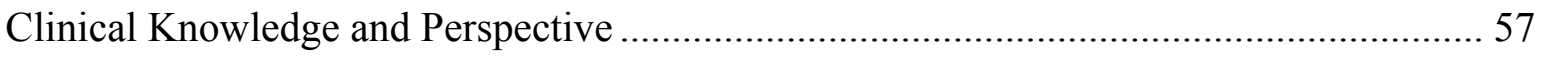

Attention to Spectrum of Mental Health......................................................................... 58

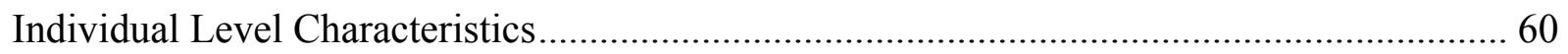

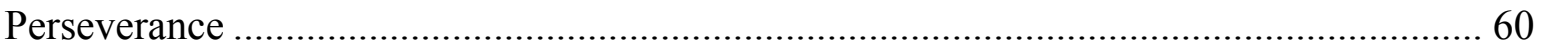

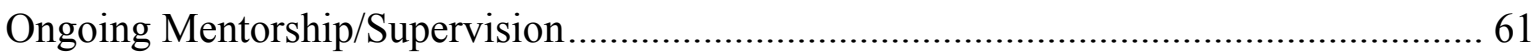

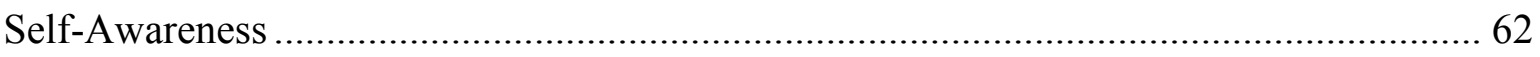

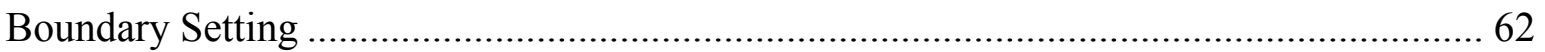

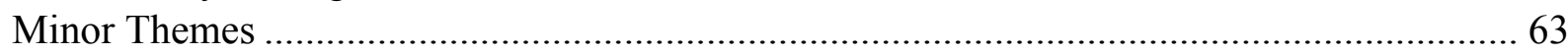

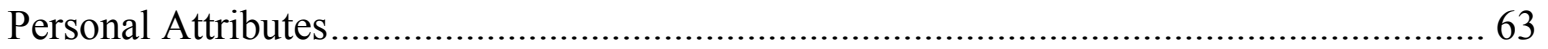

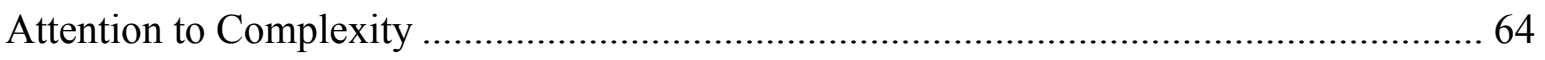

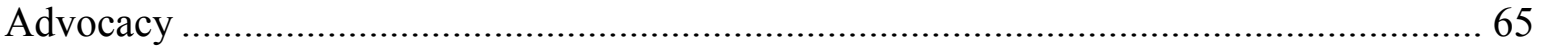

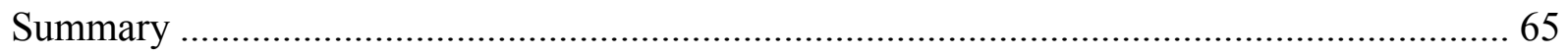

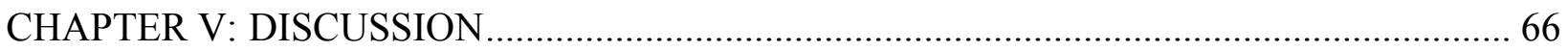

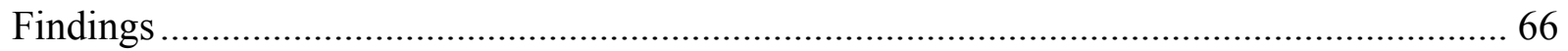

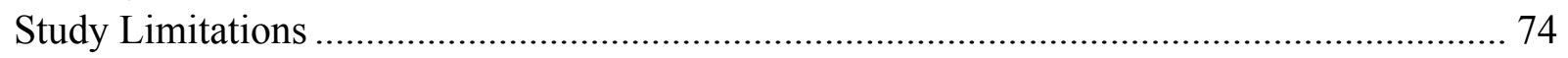

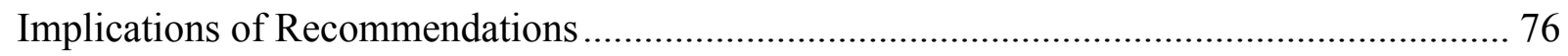

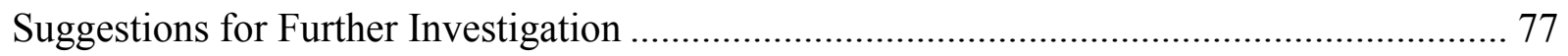

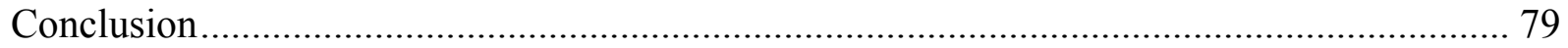

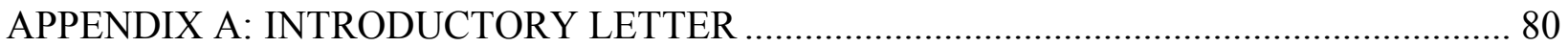

APPENDIX B: CONSENT TO PARTICIPATE IN RESEARCH ...................................... 82

APPENDIX C: CONSENT TO PARTICIPATE IN RESEARCH: INTERVIEW

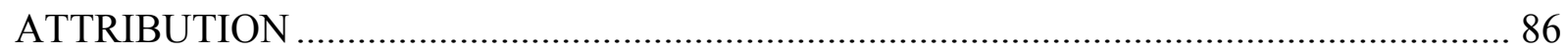

APPENDIX D: SEMI-STRUCTURED INTERVIEW QUESTIONS ................................... 88

APPENDIX E: DEMOGRAPHICS QUESTIONNAIRE ............................................... 90

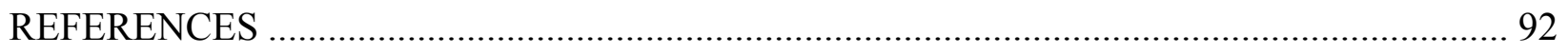

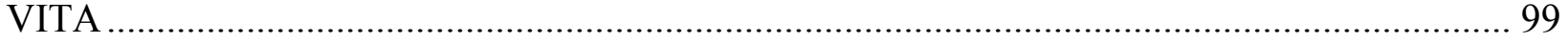




\section{LIST OF TABLES}

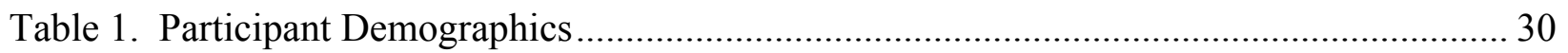

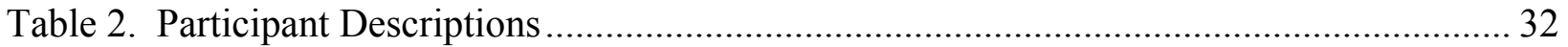

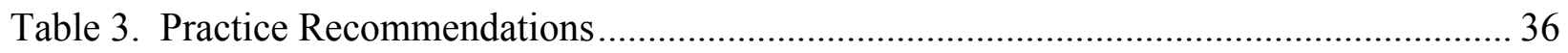




\section{CHAPTER I}

\section{INTRODUCTION}

Global mental health $(\mathrm{GMH})$ is broadly envisioned as a method of improving and achieving mental health equity for all people worldwide (Patel \& Prince, 2010). GMH attends to both mental illness as well as mental wellbeing, while also considering contextual factors (e.g., political and economic domains) and human rights issues related to mental health treatment (O'Donnell, 2012). In order to more explicitly capture the role of context in mental health, Fernando (2012) suggested a slightly modified definition of GMH described as "the mental health and mental ill health of people across the globe, experienced and expressed in culturally distinct ways" (p. 398). Overall, calls for greater attention to internationalizing the practice of psychology are in place (Forrest, 2009). Internationalization is described as the mechanism through which individuals recognize "themselves in a global context" and become aware of the "perspectives of their own specific history and culture" (Bullock, 2014, p. 6). The present study intended to examine best practice recommendations for psychologists engaged in the practice of GMH and to consider ways that the field of psychology is poised to address challenges to GMH, thereby adding unique contributions to this developing field.

\section{Growth of the Global Mental Health Field}

While GMH is a burgeoning field, attention to GMH is a growing component of psychological practice (Thornicroft, Cooper, Van Bortel, Kakuma, \& Lund, 2012). This theme is reflected in the Vision Statement of the American Psychological Association [APA] (2009), which 
confirms that the APA seeks to advance psychological science in part by acting as a "global partner" through efforts to increase knowledge and practice of psychology in diverse communities on an international level. In keeping with this goal, APA's Committee on International Relations in Psychology (CIRP) works to foster relationships within the psychological community worldwide in order to meet global challenges (APA, 2012). CIRP is also currently working to delineate competencies of professionals when working outside of the United States (Bullock, 2016).

Existing literature demonstrates significant contributions to the field of GMH by psychologists. Some of these practitioners work alongside community members to create and adapt culturally relevant interventions (Meyers, 2006). For some professionals, positive impacts from such interventions serve as a means to advocate for additional focus on creating programming for underserved communities (Bolton et al., 2003). Other projects have included efforts to better understand the impact of HIV/AIDS on women's health by collaborating with South African women to identify existing coping mechanisms and to advocate for system change, including poverty alleviation strategies that could work in tandem with mental health services (Burgess \& Campbell, 2014). Further efforts have emphasized identifying and training community members to serve as lay counselors in their communities as a means of scaling up mental health services (Murray, et al., 2011; Patel et al., 2010). Related research endeavors have investigated additional methods of providing support to youth living with HIV and called for further development of interventions that can improve the experiences of such individuals (Lypen, Lockwood, Shalabi, Harper, \& Ngugi, 2015).

Importantly, attention to mental health is a critical aspect of general global health. Global health posits the importance of defining priorities based upon disease burden, is based in 
principles of equity, and is interested in promoting health for all (Patel, 2014). Incorporation of a global mental health agenda dovetails with this agenda by working to minimize the existing burden of mental health concerns worldwide, by fighting lack of access to treatment and stigma attached to mental illness, and by developing innovative methods of delivering mental health services to global populations (Patel, 2014). Attention to mental health is a necessary step, then, in establishing a global health agenda.

Awareness of the need for GMH continues to grow. One striking factor is greater recognition of the sheer burden of mental illness. The World Health Organization (2001) reported that approximately 450 million people worldwide suffer from neuropsychiatric diagnoses, including diagnoses such as depressive disorders, schizophrenia, panic disorder, and others. While needs for treatment are high, lack of access to treatment is a recognized problem, with some estimates indicating that over three-quarters of those with serious mental illness receive little to no treatment (Becker \& Kleinman, 2012). This phenomenon is otherwise described as the mental treatment gap, which is a "proxy for the mismatch of disease burden with extant resources" (Becker \& Kleinman, 2012, p. 3).

\section{Mechanisms of Moving Towards a Global Mental Health Agenda}

While these needs for treatment are established, barriers still remain to GMH work. Some of these limitations include lack of funding for mental health treatment, centralization of resources in urban areas, and lack of mental health care workers (Saraceno et al., 2007). Stigma, need for increasing efforts from the mental health community to address GMH, and further exploration into the etiology and differing cultural manifestations of mental illness will require continued attention to advance the GMH agenda (Patel, 2012). 
Multiple suggestions exist for reducing this treatment gap. Particularly, attention to adapting and creating treatments that have a greater cultural relevance and empirical support is encouraged (Becker \& Kleinman, 2012). Capacity building, developed via partnership and longterm commitment, is also advocated as one potential mechanism to increase treatment access (Becker \& Kleinman, 2012; Fricchione, Borba, Alem, Shibre, Carney, \& Henderson, 2012). Task shifting, or mental health care delivery by lay staff, is another possible route to create access (Kakuma et al., 2011). Greater attention to research, and particularly global cooperation in research, is encouraged to share data and expertise. These practices can also serve to disseminate best practice recommendations for GMH work, with the aim of reducing the treatment gap (Collins et al., 2011; Forti, 2005; Khenti et al., 2012).

While calls for such collaboration are in place, clearly defined best practices in GMH work have not been fully articulated. Using combinations of key words international psychology, global mental health, lessons learned, best practices, and case studies resulted in a dearth of articles explicitly detailing methodology that leads to effective GMH practice. This was particularly noteworthy within the psychology literature; however, some basic recommendations appeared with the public health and psychiatry journals. For example, Khenti et al. (2012) described five factors critical to effective GMH practice, including "reciprocity, sustainability, holistic health, cultural competence, and the improvement of overall health and quality of life" (p. 90). In 2015, Khenti et al. updated these factors to include "holistic health, cultural and socioeconomic relevance, partnerships, collaborative action-based education and learning and sustainability" (p. 38). While helpful, these factors remain nonspecific to psychologists. 
On more of a structural level, attention to partnerships between organizations and individuals is one aspect of GMH that has been more fully explored in the existing literature; this attention can likely be partly attributed to suggestions that these mechanisms promote capacity building in GMH and are better equipped to address complex health problems (Fricchione, Borba, Alem, Shibre, Carney, \& Henderson, 2012; Godoy-Ruiz, Cole, Lenters, \& McKenzie, 2016). Recommendations for such partnerships, again minimal in psychology literature, are more readily available in related disciplines. Some such recommendations come from Massachusetts General Hospital Division of Global Psychiatry, which focuses exclusively on treatment, research, and capacity building to treat mental health disorders worldwide. Via their work, this institution has recommended that effective global partnerships are culturally sensitive, focused on systems change, based in evidence, clinically relevant, established as multi-year commitments, community-based, and reviewed through constant evaluation (Massachusetts General Hospital Global Psychiatry, n.d.). Forti (2005) suggested that relationships should be egalitarian and respect differences, built on mutual trust, result in increased credibility of each partner, and increase participation of each member over time. Other factors, such as conducting an initial comprehensive needs assessment, creating well-defined expectations, and incorporating flexibility and empathy, have also been encouraged (Kayingo, et al., 2016; Sapag, Herrera, Trainor, Caldera, \& Khenti, 2013).

In addition to organizational level recommendations, personal attributes of professionals engaged in GMH also are critical, though further definition of these competencies is still in its growing stages. Bullock (2016) noted that some of these characteristics include "curiosity about others, humility about the extent of one's own expertise, a nonjudgmental approach when 
observing or learning about the practices of others, and a willingness to suspend imposing one's own, familiar framework" (p. 8). Further exploration into specific competencies will be an important part of the continued evolution of GMH.

\section{Challenges to Global Mental Health}

As noted above, there are gaps in the current psychological literature examining the specific practices of competent and effective practices in global mental health work. Generally, a significant hole in the psychological literature is a minimal and non-cohesive summary of best practices in GMH, without which the potential for harm in GMH is possible. This current gap is particularly unfortunate as psychology offers a unique voice to consider some of the concerns facing the practice of GMH. There are certain areas in the practice of GMH, described below, where greater attention from the psychological community offers both insight and expertise, and further exploration from the psychological community about recommendations to address these concerns would aid the expansion of GMH efforts.

One area that is ripe for further exploration involves the process through which an individual engages in international work. Namely, what are both individual factors that are necessary for competent practice and lessons learned through the process of internationalization? Bullock (2014) suggested that a critical first step in this process is for individuals to first examine one's own cultural assumptions that influence how psychological phenomenon are understood. She noted that this process can often be quite challenging, as it requires individuals to consider issues of power and privilege, to recognize that there is much diversity in human behavior and in varying conceptions of "normal" or "healthy" mental well-being, and to accept that interventions do not necessarily seamlessly transition from cultural context to another (Bullock, 2014). One 
example of this issue can be seen even in global surveys of rates of mental illness, as most projects do not adequately address cultural beliefs about mental illness but rather apply outside models of psychopathology to varying cultures arbitrarily (Fernando, 2012). In other words, are rates of mental illness accurately captured when Western models of psychopathology are applied globally? Do these surveys both capture psychological distress and distinguish true distress from variations in behavior? While these are very large questions to address, a fundamental starting point begins with awareness of the role that context and culture play in mental health. There is little information, particularly within the literature related to $\mathrm{GMH}$, that explores the process through which professionals learn to adjust their cultural lenses in GMH practice.

Others within the GMH community fear that there is not enough attention focused on the incorporation of social determinants of mental health into interventions (e.g., Fernando, 2012; Rosso Buckton, 2015). In part, these criticisms stem from challenges and potential errors that can occur from attempting to apply Western beliefs about psychopathology and effective psychological interactions in non-Western cultures (Kidd et al., 2016; Kirmayer \& Pedersen, 2014). For example, a lack of understanding and recommendations about ethical interventions on a global scale might lead professionals to only consider individual internal experiences of mental health. Such a perspective is narrow, and a wider scope that consider individual's within the context of community, remembering that the sum of community distress might be greater than individual stress, might result in efforts that are more culturally specific (Fernando, 2012). While guidelines are growing in related areas of psychology, a cohesive set of recommendations about how to more effectively incorporate culture and communities in GMH practice would add value and likely minimize harm (Kirmayer \& Pedersen, 2014). Though the field of GMH clearly 
articulates the foundational necessity of attending simultaneously to both biological aspects of mental health and sociological determinants of mental health in order to yield truly successful interventions (Patel, 2014), further guidelines on actually applying this competency are needed. Psychology, and particularly counseling psychology, with is its attention to social and cultural factors that impact mental health, potentially offers much benefit to this conversation.

A different criticism of GMH is its potential to perpetuate power differentials and to minimize considerations of social justice. Patel (2014) called for a GMH agenda that is rooted by an interdisciplinary approach addresses mental health inequalities via cooperation with community groups that are governed by a strong social justice orientation. Patel (2014) stated:

It would be fair to say that the defining characteristic of global mental health research is that it is carried out with great attention to context and culture and by investigators with a profound understanding of the setting of their research and compassion for their "subjects." One of the unique aspects of global mental health is the extent of engagement with communities and acknowledgment of context in the design, implementation, evaluation, and uptake of research. (p. 783)

The challenge comes from taking this "rhetoric to reality" (Campbell \& Burgess, 2012, p. 391). How do professionals engaged in GMH truly collaborate with communities and advocate for the voices of community experts? The necessity of bolstering local communities to feel empowered to have a voice in setting agendas and acting as change agents is encouraged, as is greater attention to ways professionals can work within already functioning community systems (Campbell \& Burgess, 2012; Kirmayer \& Pedersen, 2014; Fernando, 2012); however, detailing of efforts to advance this practice are needed. Finally, a similar point of consideration involves the idea that GMH promotes medical imperialism, an imbalance stemming from a flow of knowledge from high-income countries to low- and middle-income countries. A suggestion to combat this concern is embracing pluralism in treatment, or in this case, greater recognition of 
community supports that are already in place to bolster mental well-being (White, Jain, \& Giurgi-Oncu, 2014). Again, specific information about how to effectively put this suggestion into practice is yet to be established.

\section{Grounded Theory}

This project utilized grounded theory, a qualitative research methodology, to explore recommendations for best practices in GMH that stem from the past experiences of identified experts in the field. Grounded theory offers a relevant framework to explore variables that have not been well explored in the existing literature (Morrow, 2007). Thus, this approach was wellaligned with the goals of this project.

\section{Purpose}

This study proposed to add to the literature on GMH by qualitatively exploring best practice recommendations for psychologists engaged in the domain of GMH. As this is a burgeoning field in mental health, consolidated efforts to explore these guidelines are still in early stages, and there are multiple holes in the literature to elucidate these processes.

Specifically, this study sought to examine the following research questions:

1. What do psychologists engaged in international health efforts consider to be essential components of "best practice" when it comes to designing and implementing successful projects?

a. What successes, challenges and struggles come in the practice of GMH?

b. How do psychologists who lead international projects view the importance of reciprocity, sustainability, holistic health, cultural competence, and improvement of quality of life in the development and implementation of their work? 
c. How can psychologists uniquely contribute to addressing health disparities and other challenges of global mental health?

This study served as an important contribution to the field of psychology, as it provides more thorough and detailed recommendations for professionals engaging in the practice of GMH. As particularly related to the field of counseling psychology, this work integrated the recommendations for culturally competent practice and the perspective of a social justice lens to consider unique issues that arise in GMH work. In doing so, this work addressed some of the challenges that are currently posed to GMH, in order to create practice efforts that both most effective, ethically-based, and culturally relevant. The next chapter will discuss historical beginnings of GMH and the current literature on the practice of GMH, including an overview of existing recommendations for global partnerships from related professions and concerns about the applied efforts of GMH work. 


\section{CHAPTER II}

\section{LITERATURE REVIEW}

Globally, four hundred and fifty million people are diagnosed with various forms of mental illness, and mental and behavioral disorders account for $12 \%$ of the global burden of disease (World Health Organization, 2001). Additionally, mental illnesses are a leading cause of disability, such that in 2017 , disability was named as the overall greatest contributor to disability, and anxiety disorders were ranked as the sixth cause (World Health Organization, 2017). While these numbers are vast, funding for mental health treatment is low, particularly in low and middle-income countries (LMICs). Specifically in low-income countries, spending on mental health is equivalent to 25 United States cents per person, per year (World Health Organization, 2011). This further impacts numbers of treatment providers, such that there is a median rate of 0.05 psychiatrists (per 100,000 population) in low income countries, 0.54 in lower-middle income countries, and 2.03 in upper-middle income countries, as compared to 8.59 in high income countries; in sum, approximately half of the global population lives in a country where there is one psychiatrist or less to serve 200,000 people (World Health Organization, 2011). Nevertheless, medications and therapies to treat these disorders are available and have the potential to be delivered to individuals and communities via alternative methods (e.g., task shifting) (Patel \& Prince, 2010). Recognition of the sheer burden of mental illness on a worldwide scale, as well as the reality of possible dissemination of treatment, is a strong impetus for the ongoing development of the field of GMH. 
A relatively new discipline within psychology, GMH developed within the past fifteen years to meet the needs described above and stems in part from the World Health Organization's global health focus (Verdeli, 2016). Global health is described as an "area for study, research, and practice that places a priority on improving health and achieving equity in health for all people worldwide" (Koplan et al., 2009, p. 1995). Patel and Prince (2010), in their influential article, note that GMH maintains this same broad focus with particular application to mental health care.

More specifically, GMH focuses both on prevention and treatment of mental health needs in the global community via interdisciplinary collaborations (O'Donnell, 2012; Verdeli, 2016). It further emphasizes attention to both individual and systems level domains, such that improvements in global health benefit the entirety of global populations and yet do not neglect the needs of individuals (Forti, 2005; O’Donnell, 2012). Lastly, GMH maintains a core focus on improving human rights of individuals with mental health disorders and mental health treatment equity (O’Donnell, 2012). This is of particular importance due to the recognition that poverty and inequality put underserved communities at greater risk, which is compounded by an inequitable distribution of resources for mental health care in low and middle income countries (LMICs) (Becker \& Kleinman, 2012; Patel \& Prince, 2010; Verdeli, 2016). This lack of resources between health care needs and resources is described as the resource or treatment gap (Becker \& Kleinman, 2012; Patel \& Prince, 2010). Upwards of 75\% of individuals with mental health disorders in LMICs receive no mental health care (WHO World Mental Health Survey Consortium, 2004), resulting in this treatment gap described as a "major failure in global health delivery" (Becker \& Kleinman, 2012, p. 3). Calls for increased engagement from the mental health community to engage with the GMH agenda are well-established (Patel, 2012). 


\section{Advancing a GMH Agenda}

There are a variety of existing recommendations of mechanisms through which to accomplish a GMH agenda. Overall, needs for scaling up mental health services are clear and are likely best accomplished by integrating mental health treatment into existing primary care services; further, development of community based care and utilization of task shifting models (i.e., training of lay community workers to provide basic mental health treatments after training and supervision) offer promise (Kakuma et al., 2011; Patel \& Prince, 2010). These mechanisms lead to capacity building, or enhanced abilities to meet mental healthcare needs through growth of a trained workforce, which is a known goal of GMH (e.g., Collins et al., 2011). Some suggest that this goal will be met only via long-term partnerships between high-income and low-income countries (Becker \& Kleinman, 2012), though critically such work must be characterized by an equal, "bidirectional" exchange of knowledge between all countries involved in such efforts (Fricchione et al., 2012, p. 54; Patel \& Prince, 2010).

GMH, despite criticisms which will be discussed later, pays explicit attention to cultural, community, and individual factors that impact mental health. This process has been described as "adapting such efforts locally... while harnessing global knowledge and experience" (Fricchione et al., 2012, p. 54). It critically notes the importance of adapting treatments that are both grounded in research and are culturally relevant (Becker \& Kleinman, 2012), while also recognizing that practitioners still have much to learn about the etiology of mental health and different cultural manifestations of mental illness (Patel, 2012). Exploration of these factors should also attend to determinants of mental illness, as well as risk and protective factors, in order to also incorporate prevention and early intervention into the agenda (Collins et al., 2011). 
Finally, GMH, with its emphasis on human rights and on challenging inequality, also clearly focuses on the need of challenging stigma for individuals diagnosed with mental health disorders (e.g., Collins et al., 2011; Patel, 2012). In addition to community-level interventions targeted to combat stigma, mental health professionals must also serve as advocates for those diagnosed with mental illness (Patel, 2012). In sum, these mechanisms are the broad goals of GMH, and accomplishing these tasks will allow for "improved care and outcomes and reduced inequities in all world regions (Patel \& Prince, 2010, p. 1977).

\section{Critiques of the Field}

As the field of GMH continues to develop, attention to ongoing critiques deserves further merit and has likely implications for recommendations regarding competent practice. Generally, these criticisms focus around the following areas: lack of attention to cultural and contextual factors, lack of attention to social inequalities, and difficulty extrapolating Western interventions to diverse communities. Further discussion of each commentary will be provided below.

\section{Lack of Attention to Cultural and Contextual Factors}

Major contributors to GMH clearly discuss the critical value of attending to cultural and contextual factors when assessing, designing, and implementing projects. For example, Patel (2014), a leading expert in GMH, explicitly states that "cultural and contextual factors profoundly influence all aspects of the mental illness experience, from its aetiology, to its expression, to the kind of help sought and the outcomes achieved" (p. 785); at the same time, this author also reflected on the need to balance the role that these variables play with integrating and providing gains from scientific advances into GMH projects (e.g., advances in knowledge of biology and medicine). Multiple authors similarly reflect on the need to find the synthesis 
between these factors, often described in terms of a local-global balance (Campbell \& Burgess, 2012; Kidd et al., 2016; Kirmayer \& Pedersen, 2014). Campbell and Burgess described (2012) this sentiment in the following way:

Professionals are experts in the biomedical paradigm of health and healing. Communities are experts in their own cultural paradigms, and best qualified to assess how to integrate what biomedicine can offer into the daily realities of their lives and survival strategies. It is vital that professional and community expertise are accorded equal status in such dialogue. (p. 388)

Nevertheless, additional commentaries that further explore the need for greater attention to cultural and contextual factors exist (e.g., Rosso Buckton, 2015). Some of the most noteworthy claims suggest that as GMH currently stands, it does not give enough credence to existing methods that promote community coping and/or grassroots approaches (Fernando, 2012; Kirmayer \& Pedersen, 2014). Similarly, it also suggested that GMH overly focuses on individual, intrapsychic effects of mental illness, while neglecting to attend to impacts of mental illness on community and group levels (Fernando, 2012). Responding in ways that is not responsive to community and cultural norms and thus lacks cultural relevance carries the potential for harm (Kirmayer \& Pedersen, 2014).

Possible solutions to combat these concerns include development of programs that are responsive to needs of local communities, such that mental health diagnoses and treatments are aligned with community perspectives (Campbell \& Burgess, 2012). Additionally, Fernando (2012) commented that addressing community needs and seeking outcomes identified as useful by the community are other mechanisms for action.

Finally, consideration of emic (i.e., attempting to understand constructs via the perspective of individuals in a particular culture, without concepts constructed prior to cultural 
engagement) and etic (i.e., understanding constructs via concepts from outside of a particular culture are used) comes into play in this discussion (Berry, 2013). Berry (2013) suggested that global psychology, and establishing global commonalities, is best approached via a "derived etics" approach, such that an "imposed etic approach (the use of Western psychology in other cultures), followed by an emic search for local phenomena... and finally the use of derived etic approach to create a global psychology that is valid for that concept or topic" (p. 59). Such an approach has great relevance in moving towards the local-global balance.

\section{Lack of Attention to Social Inequalities}

Further concerns with the GMH agenda include its lack of attention to social inequalities that affect and perpetuate mental health concerns. Campbell and Burgess (2012) described a "disconnect" between the identification of the role of systemic inequalities, such as poverty, and the current solutions proposed within the GMH community; similarly as suggested above, these authors suggested that the GMH community needs to broaden its focus beyond emphasizing individual level treatment and advocacy of human rights (p. 381). Considerations regarding power also follow under this category, particularly the role that power plays in perpetuating inequalities and need for greater attention in GMH regarding power differentials inherent in partnerships (Campbell \& Burgess, 2012; Fernando, 2012; Rosso Buckton, 2015). As an example of this premise, the majority of the priorities established in the GMH agenda are founded in and by high-income countries (Kirmayer \& Pedersen, 2014).

In response to these concerns, possible improvements include authentic partnering of researchers and community members, such that communities are identified as experts of 
themselves (Campbell \& Burgess, 2012). Mental health professionals are also cautioned to not impose their frameworks onto communities, but instead build off of existing strengths inherent in communities and identify areas of health (Campbell \& Burgess, 2012). Lastly, acknowledging and attending to power differentials is also vital, particularly as feelings of inadequacy may keep community members silent from voicing perspectives regarding community needs and program adaptions (Campbell \& Burgess, 2012).

Of note is the response from the GMH community, which posits the need for balance between addressing social concerns and individual, biomedical needs concurrently and with attention to both sets of factors; in this way, there is attention both to prevention of illness and treatment of illness that is already present (Patel, 2014). Patel (2014) noted that while attending to social variables is a critical variable of GMH, disregarding the individual and the "role of health care because of this association would be tantamount to telling a woman whose arm has been broken by her violent husband that she should approach political leaders to sort out gender inequalities rather than fixing her arm" (p. 782).

\section{Difficulty Extrapolating Western Interventions to Diverse Communities}

The final category of criticism related to the GMH agenda centers on the role of Western psychological diagnostics and interventions and their applicability to global communities. Some authors have suggested that common diagnostic guidelines (i.e., Diagnostic and Statistical Manual of Mental Disorders and International Classification of Diseases) lack validity outside of Western populations (e.g., Kirmayer \& Pedersen, 2014; Summerfield, 2008). For example, Summerfield (2008) pointed to changes in diagnostic categories that occur over time due to lack of "straightforward biological causes," suggesting that social and cultural trends instead 
influence modern understanding of mental health and illness (Summerfield, 2008, p. 992). As a corollary, if diagnostic categories lack cross-cultural merit, concern over the applicability and useful of Western interventions in non-Western settings becomes problematic. Nevertheless, some suggest that the standard practice of GMH is to utilize traditional evidence-based practices without much adaptation (Kirmayer \& Pedersen, 2014). Further, Summerfield (2008) asked if "psychiatric approaches honed in relatively well resourced and stable societies distinguish mental disorder from normal responses to a social world that is no longer coherent or functional" (p. 993).

These are significant concerns that necessitate close attention and careful consideration. Suggestions for improvement include focusing on local concepts that define mental illness as a critical starting point in terms of developing appropriate screening and intervention (Summerfield, 2008). Further, adapting interventions beyond surface level changes is likely important, such that deep adaptations to interventions account for cultural and historical aspects of societies that influence mental illness (Castro, Barrera, \& Holleran Steiker, 2010).

Within the GMH community, these concerns are addressed by Patel (2014), who countered that requiring an evidenced biological cause of mental illness to assume commonality is akin to a perspective such that "tuberculosis could not be considered a disorder till Koch discovered the bacillus responsible for it, and dementia was not a disorder (indeed, it was often seen as simply growing old badly) until its defining neuropathological features were identified" (p. 781). Patel (2014) continued to challenge this argument by noting that GMH interventions are more typically the result of "years of mixed-methods research," involving cultural adaption of research instruments and interventions and inclusion of individual (p. 783). 


\section{Existing Recommendations for Practice}

In light of these concerns, further delineation of best practice are needed to ensure that GMH continues to advance an agenda that is ethical, grounded in clinical knowledge, and responsive to community needs. Significantly, establishing GMH best practices has the potential to buffer concerns discussed above and add additional credence to existing rebuttals.

One particular area of GMH that is ripe for further exploration relates to best practice recommendations that are specific to psychologists who engage in such work. Notably, while other domains, including public health and psychiatry, have recommendations in place for practitioners, the field of psychology currently has a gap in its existing literature to guide research and clinical practice in GMH. While recommendations for specific settings are in place (e.g., humanitarian settings; see Tol et al., 2011), guidelines for general practice are understudied. Nevertheless, as these recommendations from other fields potentially have overlap with recommendations for psychologists, the extant literature base will next be reviewed.

Of note is the Holistic Policy and Intervention Framework for global mental health, (HPIF), which represents an effort from the Office of Transformative Global Health at the Centre for Addiction and Mental Health (CAMH), the largest organization dedicated to mental health and addiction in Canada (Khenti et al., 2015). This model is described as an approach that "can guide decision makers and health-care professionals in capacity building in global mental health" and stems from a "decade of collaborative initiatives and the lessons learned, as well as in existing global health best practices" (Khenti et al., 2015, p. 38). The "five pillars" inherent in this model include attention to "holistic health; cultural and socioeconomic relevance; partnerships; collaborative action-based education and learning; and sustainability" (Khenti et 
al., 2015, p. 38); the former domains described in Khenti et al. (2012), including "reciprocity, sustainability, holistic health, cultural competence, and the improvement of overall health and quality of life" (p. 90) were integrated into this later work. Many of these themes are reflected more broadly in the global health literature, and each factor will be described in detail below.

\section{Holistic Health}

As described in this model, holistic health refers to the necessity of understanding the context of individuals and communicates via expanding beyond a biomedical lens, while also attending to relationships between individuals and larger systems (e.g., policies, existing stigma, lack of resources for mental health treatment) (Khenti et al., 2015). Beyond simply recognizing these larger systems, attention to bolstering efforts and implementing changes to support mental health at various systems levels is also advocated (Khenti et al., 2015; Sapag et al., 2013). For example, aims of improving mental health treatment and care need to include efforts that strength economic and social improvements, such as improvements in access to housing and education (Wallcrat et al., 2011).

On a related note, attention to stigma as a barrier to care is another component of holistic health (Khenti et al., 2015) and has also been identified as major barrier and necessary aim of the GMH agenda (Patel, 2012). Stigma exists on multiple levels, including both individual and structural levels (Kidd et al., 2016). Moreover, multiple authors (e.g., Ravitz et al., 2014; Wallcrat et al., 2011) discussed decreasing stigma of mental illness in health care providers as an important aim.

\section{Cultural and Socioeconomic Relevance}

Next, Khenti et al. (2015) described the importance of cultural and socioeconomic relevance as part of their framework, describing this as a bottom-up approach that allows for 
perspectives and knowledge of mental health to originate from individuals and communities, such that GMH are relevant and meaningful for local communities and not prescribed by outside professionals. Using this approach, efforts are designed to work towards symptom reductions in ways that are responsive to both local community expressed needs and broader commonly accepted needs (Shah, 2011). In addition to this emphasis existing within the GMH agenda described earlier in this paper, this same recommendation is broadly espoused in the literature. For example, Ravitz et al. (2014) noted that understanding of context is a critical factor that affects implementation and policy changes, and other authors have discussed the necessity of prioritizing local knowledge to create relevant, effective treatments (e.g., Raviola, Eustache, Oswald, \& Belkin, 2012; Sapag et al., 2013). Involving stakeholders, both in the general community and also service users themselves, is a necessary path through which to meet this aim (Boutilier, Daibes, \& Di Ruggiero, 2011; Khenti et al., 2015; Shah, 2011; Wallcrat et al., 2011), best accomplished by undertaking a thorough needs assessment (Sapag et al., 2013; van Ommeren, Saxena, \& Saraceno, 2005). Lastly, expanding beyond the community level, Patel and Prince (2010) similarly described approaches built upon a birdirectional exchange of knowledge between high income and LMICs.

Moreover, accounting for cultural and socioeconomic relevance is manifested through attitudes that include "respect, openness, and humility" (Khenti et al., 2015, p. 41). Other authors similarly echo this perspective, with particular emphasis on respect, trust, and flexibility emerging as factors that lead to successful efforts (Forti, 2005; Kayingo et al., 2016; Shah, 2011; Tribe, Weerasinghe, \& Parameswaran, 2014). Foundationally, respect for human rights is a core component of successful partnerships (Wallcrat et al., 2011). 
Partnerships

In the context of global health, partnerships are described as "contextually relevant peerto-peer collaborations which offer a platform for sharing knowledge and growing expertise globally, working towards a common goal, across disciplines and perspectives" (Larkan, Uduma, Lawal, \& van Bavel, 2016, paragraph 2). As stated by Forti (2005), partnerships in global health are understood as mechanisms that involve individuals and broader societal structures, that respect diversity, that rely upon shared responsibility between members, that seek equality in health provision, and that lead to autonomy and capacity building (p. 32). In response to these definitions, existing recommendations focus both on broad, systemic, overarching themes that are descriptive of healthy partnerships, as well as more specific, individual actions and traits that are equally necessary. Partnerships are increasingly seen as the mechanisms to address complex health problems that require more intensive, multi-focused interventions (Godoy-Ruiz et al., 2016).

Reciprocity is another critical variable of effective partnerships (Khenti et al., 2015; Khenti et al., 2012), in part recognized through mutual benefit of projects for all stakeholders (Larkan et al., 2016). While reciprocity is often espoused as the goal of collaborative endeavors, multiple authors have discussed the challenge of this goal in practice, due to tendencies of highincome countries (HICs) to view LMICs as beneficiaries and recipients of knowledge instead of as equal partners (e.g., White, Jain, \& Giurgi-Oncu, 2014). Instead, reciprocity is better structured to promote "counterflows," described as an exchange of knowledge and benefit so that ideas from LMICs in turn influence mental health practice HICs (White, Jain, \& Giurgi-Oncu, 2014, p. 602). Elsewhere, this concept is described as "bidirectionality," which similarly 
suggests that all collaborating members have opportunities to benefit and gain from partnerships (Fricchione et al., 2012, p. 54). Steps towards reciprocity include a focus on building equity in partnerships from the developmental to evaluation stages of collaborations (Godoy-Ruiz et al., 2016; Raviola et al., 2012).

The critical role of ongoing communication as a tool for partnership building is highlighted repeatedly (e.g., Forti, 2005; Kayingo et al., 2016; Khenti et al., 2015; Larkan, et al., 2016). In addition, the value of setting and revisiting expectations throughout collaborations is a common theme (Kayingo et al., 2016; Wallcrat et al., 2011). Such expectations might relate to the structure of the research team, plans for authorship and publication, and extent of the research project; clarity of such expectations is a critical mechanism to promote clarity and to prevent discrepancy other team members (Forti, 2005; Kayingo et al., 2016; Sapag et al., 2013). Kayingo et al. (2016) further suggested that communication is sensitive to cultural differences in order to best promote responsive, respectful partnerships. Similarly, ongoing evaluation that involves all stakeholders comprises another critical aspect of open communication (Sapag et al., 2013; van Ommeren, Saxena, \& Saraceno, 2005). Some authors comment that involving all stakeholders in such planning and evaluation stages is insufficient, due to power differentials that favor professionals; most effective communication is enhanced by creating environments that are receptive to local community opinions, evidenced by powerful stakeholders valuing and critically listening to needs brought forth by local communities (Campbell \& Burgess, 2012).

\section{Collaborative Action-Based Education and Learning}

Khenti et al. (2015) also described the importance of working in partnerships to identify areas of strength and growth in targeted communities and provide education that examines 
tangible problems. General discussions of the importance of collaborative partnerships are discussed at length in the existing literature (e.g., García-Ramírez, Paloma, Suarez-Balcazar, \& Balcazar, 2009; Godoy-Ruiz et al., 2016), with suggestions that collaborative approaches result in improved learning and a greater sense of efficacy for partners (García-Ramírez et al., 2009).

\section{Sustainability}

Finally, Khenti et al. (2015) describe sustainability as the final pillar of their approach, which stem in part from the reciprocal relationships and capacity building efforts described above; necessarily, sustainability is only meaningful such that it is reflective of context-specific needs and is driven by community ownership. Thus, partnerships are most effective when designed to promote sustainability (Forti, 2005; Raviola et al., 2012; Sapag, Herrera, Trainor, Caldera, \& Khenti, 2013; Shah, 2011; van Ommeren, Saxena, \& Saraceno, 2005).

Outside of these recommendations from Khenti et al. (2015), a few additional factors deserve further attention. Namely, some authors have posited the importance of involving interdisciplinary teams (Boutilier et al., 2011; Raviola et al., 2012; Sapag et al., 2013), noting that global health issues are complex and require responses from a varying disciplines. Additionally, awareness of power differential and efforts to reduce such differentials is of importance (Campbell \& Burgess, 2012; Sapag et al., 2013; Shah, 2011), in order to assist local communities with meeting expressed needs and improving community empowerment.

Outside of the themes detailed by Khenti et al. (2015) and supported by other authors, a brief review of recommendations from large global health organizations and programs will be briefly reviewed. For example, one such set of recommendations come from Massachusetts General Hospital Division of Global Psychiatry. Via their global health work, this institution has 
recommended that effective global partnerships are culturally sensitive, focused on systems change, based in evidence, clinically relevant, established as multi-year commitments, community-based, and reviewed through constant evaluation (Massachusetts General Hospital Global Psychiatry, n.d.). Similarly, the Canadian Coalition for Global Mental Health (2015), which works to promote equity in global health, suggest the importance of themes including authentic partnering, inclusion, shared benefits, commitment to the future, responsiveness to causes of inequalities, and humility. Lastly, the Commission for Research Partnerships with Developing Countries is an organization based in Switzerland that works to promote equitable research collaboration with LMICs in the efforts to eliminate global problems; their efforts have resulted in a handbook outlining considerations that underscore successful partnerships. These principles include setting the agenda together, interacting with stakeholders, clarifying responsibilities, accounting to beneficiaries, promoting mutual learning, enhancing capacities, sharing data and networks, disseminating results, pooling profits and merits, applying results, and securing outcomes (Swiss Commission for Research Partnerships with Developing Countries, 2014). Of note, while these recommendations come from a variety of sources, similar themes exist in each set of guiding principles and are also reflective of the guidelines set forth by Khenti et al. (2015).

\section{Summary}

While existing commentary regarding best practices and useful frameworks for GMH exists, such documentation specifically targeting psychologists is lacking. Further, many of these recommendations stem from large organizations and do not reflect individual perspectives or examples of how these best practices impact successful GMH projects. This literature base is 
important to the exploration of existing recommendations for GMH and provides a point from which to launch further investigation. This study interviewed expert-level psychologists active in the GMH in order to determine best practices that guide their work. 


\section{CHAPTER III}

\section{METHODS}

This chapter outlines the project's research design and also provides a rationale for using a qualitative approach for data collection and analysis. Of note, qualitative methods allow researchers to grasp a more nuanced understanding of experience, as well as offering opportunities to better explain meanings that individuals attribute to these experiences (Morrow, 2007). These approaches are also particularly useful when exploring variables that have not been well explored in the existing literature (Morrow, 2007). This project, with its focus on recommendations from best practices that stem from past experiences and on developing guidelines in an underdeveloped field, is well aligned with the goals from qualitative methods.

\section{Grounded Theory}

Grounded theory is a qualitative research design that generates theories from data grounded in the field, particularly by paying attention to the "actions, interactions, and social processes" of participants and then integrating these processes into hierarchical categories (Creswell, Hanson, Clark Plano, \& Morales, 2007, p. 249). Theory arises through the constant comparative method, which is accomplished through simultaneous analysis and data collection; this method serves to identify repeated variables under specific conditions, resulting in concepts and hypotheses (Glaser \& Holton, 2007). The resulting theory is one defined by conceptual relationships, based upon patterns and processes of interaction in the data (Strauss \& Corbin, 1994). Patterns play a particular role in grounded theory, as concepts are only interwoven into the 
resulting theory as they are found repeatedly in the data, thus guarding against researcher bias (Corbin \& Strauss, 1990). In all, results derived from grounded theory create a "substantive" theory that that has is aligned with "specific, everyday-world" situations, thereby offering theories with specificity and usefulness (Merriam, 2009, p. 30). The intention of the present study is to synthesize recommendations for best practices from experts in GMH, while also expanding upon previous research by considering the unique role the psychology offers this growing field.

\section{Methods}

\section{Participants}

Participants were required to have a doctorate degree in psychology to be eligible for this study. Additionally, participants needed to have demonstrated hands-on experience and dedicated time working abroad in a professional capacity. As this study sought to gather information from identified experts with a extensive history of scholarship, employment, and front-line practice in the field of GMH, while also specifically investigating unique contributions from psychologists, this rigid criteria was necessary to ensure that information provided by participants was grounded in both appropriate education and experience.

Qualitative approaches use a purposeful sampling technique, such that invited participants meet specific criteria guidelines in order to provide rich data (Morrow, 2005). Participants for this study were initially recruited through identification of significant contributions to the existing literature on GMH and subsequently through a peer nomination system from initially identified experts.

Determination of sample size in qualitative research is not a function solely of numbers of participants but is also a reflection of adequacy of data. Nevertheless, Morrow (2005) 
suggested that approximately 12 participants is often an adequate sample size prediction. More importantly, data should be collected to the point of redundancy, or until additional information is no longer forthcoming (Morrow, 2005). For grounded theory, it is the representativeness of repeated concepts that are important, rather than number of individuals (Corbin \& Strauss, 1990).

For this study, 15 initial participants received an email (Appendix A) outlining the purpose of the study, an invitation to participate, informed consent forms (Appendices B and C), and criteria for inclusion in the project; the primary investigator later contacted eight additional individuals, and no individuals referred via peer nomination agreed to participate. Overall, nine individuals agreed to participate in this study. All participants received informed consent documents, which included information regarding the nature and scope of involvement by the individual and permission to withdraw from the study at any time without consequences; further, participants were offered the opportunity to have all or part of their interviews attributed to them, and four individuals approved interview attribution. All participants also received the interview questions (Appendix D) prior to the interview, in order to allow them to develop thoughts on their involvement tin GMH. Participants also received a demographics questionnaire (Appendix E).

Of the nine participants, six returned the demographics questionnaires. Of these, there were two males and four females. The range of ages for participants was 42 to 64 with an average of 53 years old. The median age was 52 years old. Three individuals from the participant pool identified as White (Caucasian, European American). One individual identified as Asian or Pacific Islander, and one individual identified as Mixed Latina/European American. One individual did not identify a racial background. Five of six respondents reported the United 
States of America as his or her country of origin, and the remaining individual did not indicate a country of origin.

When asked to identify their current place of employment, five of the six respondents indicated employment at a university. The remaining individual reported working for a NGO. Further, five of six respondents reported obtaining a $\mathrm{PhD}$ in clinical or counseling psychology, and the remaining individual reported receiving a PsyD in clinical psychology. Respondents indicated a range of experience from 12 to $40+$ years, as well as job duties typically comprising research, clinical, teaching, and consultation roles. Table 1 provides the demographic data for the participant pool.

Table 1. Participant Demographics

\begin{tabular}{lll}
\hline Variables & \% & n \\
\hline Age & 22 & 2 \\
$40-49$ & 22 & 2 \\
$50-59$ & 22 & 2 \\
$60-69$ & 33 & 3 \\
No response & & \\
Gender & 44 & 4 \\
Female & 22 & 2 \\
Male & 33 & 3 \\
No response & & 5 \\
Educational Level & & 1 \\
PhD & 56 & 11 \\
PsyD & & \\
\hline
\end{tabular}




\begin{tabular}{lll}
\hline Variables & $\mathbf{\%}$ & $\mathbf{n}$ \\
\hline No response & 33 & 3 \\
Country of Origin & 56 & 5 \\
United States of America & 44 & 4 \\
No response & & \\
Racial Identification & 33 & 3 \\
White (Caucasian, European American) & 0 & 0 \\
Black (African American) & 11 & 1 \\
Asian or Pacific Islander & 0 & 0 \\
Hispanic (Latino/a) & 0 & 0 \\
Native American & 11 & 1 \\
Other & 36 & 5 \\
No response & 44 & 4 \\
Current Employer & & 5 \\
University/College & &
\end{tabular}

$* \%=$ percentage of participants who responded in this category. $n=$ number of participants in each category

*Due to rounding, percentages may not add to $100 \%$. 
Table 2. Participant Descriptions

\begin{tabular}{|c|c|c|c|c|}
\hline $\begin{array}{l}\text { Participant } \\
\text { Number }\end{array}$ & Gender & Employer & $\begin{array}{l}\text { Years of GMH } \\
\text { Experience }\end{array}$ & $\begin{array}{l}\text { Type of GMH } \\
\text { Experience }\end{array}$ \\
\hline 1 & Female & University & $15+$ years & $\begin{array}{l}\text { - } \text { Research } \\
\text { - Teaching }\end{array}$ \\
\hline 2 & Female & University & $10+$ years & $\begin{array}{l}\text { - } \text { Research } \\
\text { - Teaching }\end{array}$ \\
\hline 3 & Male & NGO & $30+$ years & $\begin{array}{ll}\text { - } & \text { Research } \\
\text { - } & \text { Consultation }\end{array}$ \\
\hline 4 & Female & University & $20+$ years & $\begin{array}{l}\text { - Clinical } \\
\text { - } \quad \text { Teaching }\end{array}$ \\
\hline 5 & Female & Organization & $10+$ years & $\begin{array}{ll}\text { - } & \text { Research } \\
\text { - } & \text { Teaching } \\
\text { - } & \text { Consultation }\end{array}$ \\
\hline 6 & Male & University & $40+$ years & $\begin{array}{ll}\text { - } & \text { Research } \\
\text { - } & \text { Teaching } \\
\text { - } & \text { Training }\end{array}$ \\
\hline 7 & Female & University & $20+$ years & $\begin{array}{ll}\text { - } & \text { Research } \\
\text { - } & \text { Consultation } \\
\text { - } & \text { Teaching }\end{array}$ \\
\hline 8 & Female & University & $15+$ years & $\begin{array}{ll}\text { - } & \text { Research } \\
\text { - } & \text { Teaching } \\
\text { - } & \text { Consultation }\end{array}$ \\
\hline 9 & Female & University & $10+$ years & $\begin{array}{l}\text { - Research } \\
\text { - Teaching }\end{array}$ \\
\hline
\end{tabular}

*Note: All participants did not complete the demographics questionnaire. If the respondent did not provide information, the primary investigator completed this table based upon publicly available information.

\section{Procedures}

Participants spoke with the primary researcher who conducted individual, semistructured, audio-recorded interviews with them, which were 45 minutes to an hour and a half in 
duration. The format of semi-structured interviews was chosen for this project, as these interviews allow for common themes to be explored among participants while offering the flexibility to explore respondent answers as needed (Suzuki, Ahluwalia, Arora, \& Mattis, 2007). The semi-structured interview asked the participants to reflect on their work and experiences in the GMH field, while also asking for consideration of past successes and challenges that have come through such practice. Additionally, the semi-structured interview encouraged participants to consider existing recommendations for the practice of GMH and to respond and add to this list. During the interview, participants also discussed opportunities that psychologists have in advancing the mission of GMH.

As a part of the protocol for grounded theory, the researcher recorded notes after each interview with impressions about the content and process of the session. Information from the interviews was coded to highlight common themes that emerged across interviews. After data were condensed, critical elements of the data were shared with study participants to check for accuracy, to allow for further review, and to check for researcher bias (Morrow, 2005).

\section{Data Analytic Procedures}

Grounded theory uses the process of theoretical sampling to inform its analysis; that is, data is analyzed as it is collected, and this analysis guides subsequent information gathering (Corbin \& Strauss, 1990; Glaser \& Holton, 2007; Merriam, 2009). Following each interview, each recording was reviewed, along with the notes that the researcher had written during and immediately after the interview process. During this review, the researcher recorded thoughts on emerging themes and burgeoning hypotheses that could be further integrated into subsequent interviews. 
Coding themes began broadly and narrowed through the use of subsequently stringent coding methods utilized in grounded theory. Initial coding serves to break up the raw data and allows the researcher to group data into codes, and later, generate theory (Glaser \& Holton, 2007). Grounded theory has three phases of coding: open, axial, and selective (Merriam, 2009). Open coding is based upon identification of any data point that might be relevant to the study (Merriam, 2009). Open coding is accomplished through identification of substantive concepts within raw data, allowing for insight into the direction that the study is moving; particularly, open coding aids in saturating categories and minimizes bias or missing data (Corbin \& Strauss, 1990; Glaser \& Holton, 2007). Axial coding then groups related concepts into categories (Merriam, 2009); categories are determined in terms of "properties and dimensions, the conditions which give rise to it, the action/interaction by which it is expressed, and the consequences that result" (Corbin \& Strauss, 1990, p. 420). Finally, selective coding develops hypotheses that relate the categories to one another (Merriam, 2009). Selective coding importantly identifies the core variable, which is considered to be the element that connects the categories, accounts for the variation in the study, and offers the basis of the resulting theory (Glaser \& Holton, 2007; Merriam, 2009).

After transcribing and identifying themes contained in the individual interview, each participant received an email asking for amendments, additions, or correction of errors of these transcriptions and themes. Of the nine participants, five individuals responded to this invitation. Comments received from the respondents typically indicated agreement with the transcription and themes, as well as minor modifications to language or the addition of one or two themes. 


\section{Researcher Background, Experiences and Biases}

The researcher is doctoral student in counseling psychology who identifies herself as a European American and a United States citizen. She is committed to learning more and working towards reducing disparities in mental health on a worldwide level and has been developing this perspective over the last ten years through her volunteer, academic, and clinical work. She is drawn to using qualitative approaches in order to better understand the meanings that individuals ascribe to their experiences, while also believing that such an approach is a useful method for helping traditionally disempowered individuals gain a voice in research endeavors. In this study, she anticipated hearing from respondents descriptions of lessons learned that are culturally responsive, sustainable, and promote a social justice agenda. 


\section{CHAPTER IV}

\section{RESULTS}

This chapter describes the significant findings and content of the semi-structured interviews conducted with participants of this study. Each participant actively engaged in this research, and many expressed interest in learning about the findings of the study.

All of the participants thoroughly described their work in GMH and their resulting perspectives on best practice recommendations for fellow psychologists. While many of the respondents engage in GMH in a variety of capacities, multiple universal skills came to the fore. From these skills, three major categories emerged, with subthemes representing recommendations embedded under each category. Table 3 provides an overview of those findings.

Table 3. Practice Recommendations

\begin{tabular}{lll}
\hline Variables & $\mathbf{\%}$ & $\mathbf{n}$ \\
\hline Overarching Variables & & \\
Consideration of Cultural/Contextual Variables & 100 & 9 \\
$\quad$ Cultural Relevance & 89 & 8 \\
Cultural Competence & 89 & 8 \\
Recognizing Similarities and Differences & 67 & 6 \\
Collaboration & 89 & 8 \\
Relationship Building & 67 & 6 \\
\hline
\end{tabular}




\begin{tabular}{|c|c|c|}
\hline Variables & $\%$ & $\mathbf{n}$ \\
\hline Reciprocity & 44 & 4 \\
\hline Communication & 44 & 4 \\
\hline \multicolumn{3}{|l|}{ Program Level Characteristics } \\
\hline Sustainability & 89 & 8 \\
\hline Evaluation and Research Methods & 89 & 8 \\
\hline Flexibility & 56 & 5 \\
\hline Attention to Systems & 56 & 5 \\
\hline Multidisciplinary Teams & 44 & 4 \\
\hline Clinical Knowledge and Perspective & 44 & 4 \\
\hline Attention to Spectrum of Mental Health & 33 & 3 \\
\hline \multicolumn{3}{|l|}{ Individual Level Characteristics } \\
\hline Perseverance & 67 & 6 \\
\hline Ongoing Mentorship/Supervision & 44 & 4 \\
\hline Self-Awareness & 44 & 4 \\
\hline Boundary Setting & 22 & 2 \\
\hline \multicolumn{3}{|l|}{ Minor Themes } \\
\hline Personal Attributes & 22 & 2 \\
\hline Attention to Complexity & 22 & 2 \\
\hline Advocacy & 11 & 1 \\
\hline
\end{tabular}

*Percentages were rounded to whole numbers.

Although some variables were mentioned with greater frequency than others, the amount of time participants spent discussing practice recommendations did not necessarily correlate to that frequency. Every attempt was made to categorize each item mentioned by the participants. 
Overarching Variables

Two main categories, consideration of cultural/contextual variables and collaboration, emerged as common themes that appear to have trickle-down implications for categories within both the program and individual level characteristics sections. Further exploration and discussion of the subthemes contained within these two overarching variables will be expanded upon below.

\section{Consideration of Cultural/Contextual Variables}

Significantly, all nine participants referenced concepts related to consideration of cultural and contextual variables. In particular, these ideas centered on development of cultural competence and awareness of mechanisms through which to create projects that are meaningful to local communities. Additionally, learning to recognize global similarities, as well as accounting for specific differences, was discussed by several participants. Each subtheme will be explored in the following sections.

Cultural relevance. Crafting GMH programs that have meaning and value in identified communities emerged as a common theme, with eight participants identifying varying aspects of this concept critical in their work. Generally, comments focused on responding to community needs, following leadership of local colleagues, and modification of interventions. As a starting point, three respondents discussed the importance of first ascertaining if proposed projects are needed or are priorities in local communities. For example, Participant \#1 described this idea by stating that "frankly if what they do works fine, and is effective, we don't have any place to do something." Other respondents expanded on this idea.

We first want to connect with them - the local population, to find out what the problems are, what already exists, what role can we fill, etc. I think the local implementing partner has a lot to do with that. I mean, we're in and out of all these countries, so I think they do 
so much of the work of being there and understanding and meeting people through a project's duration. (Participant \#8)

I was more interested in ensuring that the way they [GMH community] decided to work was in line with what people were actually asking for [in terms of treatment]. (Participant \#9)

As referenced by the participants above, following the guidance of local colleagues to craft culturally relevant programs is vital. Repeatedly, other participants underscored the importance of this concept and its application in their work, as well as considered how working alongside local partners directly impacts adaptation and implementation of projects.

I'd say that especially with the local teams that guide us on how to adapt, how to select various strategies, how to adapt things to make them locally relevant. We follow our local colleagues' guidance. And specific adaptions of manuals, for example. And training and techniques and implementation. So that's collaboration and engagement and really understanding what our local colleagues are telling us. And use it. (Participant \#1)

I have really good local colleagues who can step in.... I think the local leadership can help figure out options for turning it all over. I think the big thing is who your broader local network is. And certainly having their support and presence is big in adjusting any of the challenges that you may run into. (Participant \#5)

If a program or project is going to be sustained and effective, the local people, the local stakeholders - whoever they might be - have to have an investment in it. They need to feel like it's theirs. We need them to help us understand the norms, help us understand how things happen, and to help us with the content. So they take ownership of it. (L. Gerstein, personal communication, March 3, 2017)

So when we work with our colleagues [abroad] in the integration of mental health and primary care, we work with the people on the ground and talk about what has worked in this protocol before and get their expertise on what will or won't work, and then modify to the extent necessary so that the intervention will have legitimacy and validity locally. So that's how we do everything, and it makes a huge difference. (K. Pike, personal communication, March 10, 2017)

In addition, program modifications that are culturally appropriate and relevant require accounting for "culture, setting, gender," as well as using a common language that does not 
stigmatize mental illness but "will be locally acceptable and will convey to the person that we're talking about this specific cluster or things that affect functioning” (Participant \#1).

Lastly, working with individuals and communities to determine and deliver relevant programs serves to build agency and equalize power in crafting projects.

So for me to say this paradigm needs dismantling completely also takes agency from people who might choose to come from this buffet of sorts of treatment and be empowered to choose for themselves what works and what doesn't work. So our job as practitioners is to ensure that services are delivered in a certain way so that agency is enabled... the way to protect this middle ground is to drive forward agendas of community participation. (R. Burgess, personal communication, March 17, 2017)

Thus, partnering to determine relevance has positive effects not only in terms of the specific intervention, but also as related to perceived self-efficacy.

Cultural competence. Working towards developing cultural competence was a necessary component of GMH identified by eight of the participants. One participant discussed the need of both recognizing cultural differences but also more broadly appreciating contextual differences, with the understanding the developing competence spans both of these variables.

Understanding context. Culture is so generic. It's understanding the context of the setting. The setting is a culture in itself, if it's primary care versus community versus a refugee camp. Understanding how to adapt for culture but also logistical/delivery fit. (Participant \#1)

In response, multiple respondents discussed problems that can arise if practitioners attempt projects without cultivating this foundational skill.

I see well-meaning colleagues without a cross-cultural background who want to have an impact... and it's a bit of a stretch to be able to help in a way that's relevant by only taking your US or European skill sets over. So I think people are being more and more aware of this... but still a lot of our defaults are to go with what's culturally familiar. I think that we need to push ourselves and work under others who are a few steps ahead of us cross-culturally. (K. O’Donnell, personal communication, February 13, 2017) 
There's no question that people need to respect the ideas around culture and have an understanding of cultural values and similarities and differences. And [they need] to know how to operate in a culture... and this is really, really important - really important - you can't work without an appreciation for the fact that when we work in global health and particularly global mental health, people are exposing themselves and there's a vulnerability associated with that kind of engagement. To not be culturally competent is a huge problem - it's not ok.” (K. Pike, personal communication, March 10, 2017)

To avert difficulties from lack of competence, five individuals identified gaining knowledge as a necessary task within working towards cultural competence. Several participants discussed this idea in the context of cultural immersion and living within different contexts.

...talking to as many people in the country that you can about perceptions and procedures there. Really immersing yourself in the culture. (Participant \#2)

It's almost like get your vaccinations, and you do it, but you don't really know until you get there that you're going to be eaten alive by mosquitos. Someone might have told me before I went to [country] 'well, that's not going to work'... But until I got there and went to that first therapy session I was doing with that family and it was a total bomb, I probably wouldn't have made sense of it anyways. It doesn't register. You don't hear it. (Participant \#5)

Living there further strengthened my relationships with the people there that I was already working with. It gave me a much deeper understanding and respect for the culture there. (L. Gerstein, personal communication, March 3, 2017)

However, gaining knowledge via cultural immersion and/or living in different contexts is mediated by variables such as respect (identified by four participants) and humility (identified by five participants). Particularly, respect is reflected in appreciation for differing norms, values and beliefs, as well as a willingness to learn about such differences.

Within that collaboration, listen to the voices of the people within the community. And have compassion, too. Sometimes the worldview of those people may not be yours. (Participant \#4)

I suppose when I look at what my psychologists successful in international settings - or anyone - is perspective; it's the way you approach that (i.e., differences between cultures)... so willingness to learn is important. (Participant \#5) 
The most important thing is respect and being able to appreciate how my colleagues overseas... go about doing their work. Not trying to infuse a US-centric point of view into the way they do things. (L. Gerstein, personal communication, March 3, 2017)

You need to spend a lot of time having tea and building trust. If you jump right in American style... 'hello, how are you, and let's start', you could never do that... we're very task oriented, and that's culturally very consistent... So you need to understand there are these cultural differences and norms, and spending the time to get it right at the beginning is the only way you're going to have a successful collaboration. (K. Pike, personal communication, March 10, 2017)

I think that's why it's so important to work with local people who understand their local cultures. And to be really respectful and know that here I am the outsider. (Participant \#8)

As an example, one participant spoke of adaptability and flexibility as a means to be

responsive to differing norms.

It requires cultural adaptability, which means you have to be able to go with the flow. In some places in the world where I do work, it really is an issue - the perspective of time is critical. In the United States, we're on some sort of a schedule to get this done. And in many places in the world, they'll say they're on a schedule, but they act really differently. The knee jerk reaction is to get upset and try to hold them to the fire, and it doesn't work. So you have to be able to let go what your current cultural values are here (i.e., in the US). You have to work with it in a way that's affirming and not demeaning to the person that you're collaborating with." (L. Gerstein, personal communication, March 3, 2017)

Further, regarding humility, participants described approaching partnerships such that

knowledge of one's limits and importance of hearing the perspective of others is evident.

With all of these I'm trying to think carefully about if it comes from the people, if it comes from the collaboration, great. But if not, I don't know that you should set anything other than cultural humility as an agenda. (Participant \#2)

And humility - to be able to recognize the limits of your own knowledge and skills and the contributions of other knowledge and skills. And I also think to be ok saying 'I don't know what to say' or 'I didn't really get that'. You can't go in with pride. (Participant \#5) 
In sum, working towards cultural competence, a critical aim in best practices, is an ongoing task assisted by supervision and mentorship, gaining knowledge, and developing respect and cultural humility.

Recognizing similarities and differences. Six participants discussed the task of learning to identify both similarities and differences, particularly in terms of human experience and manifestations of mental illness.

It's very strange, but at the same time but I'm aware at the same time how different and similar people are. We find some differences, of course, in expression of depression - I mean we call it depression - but expression/state of resignation or hopelessness and helplessness and reactions to loss. So I think the similarities actually may outweigh the differences. But it's very important to understand the differences. (Participant \#1)

That is absolutely the case that there are cultural differences in the expression of certain mental health conditions and cultural differences in terms of health systems and ideas around etiology. But there's also an enormous commonly shared platform. And the unique disorders that exist in various parts of the world exist in addition to the globally recognized nature of mental illnesses, like schizophrenia and other forms of psychosis and depression and anxiety and eating disorders. So I think there is a lot we can do by bringing together a global network. (K. Pike, personal communication, March 10, 2017)

Finding the balance, then, by both accounting for differences while recognizing commonalities, was referenced as a critical skill.

Further, utilizing and adapting existing psychological treatments potentially serves to meet the expressed needs of individuals targeted by GMH projects and requires a fundamental understanding of the interplay of these factors. However, one respondent cautioned against simply assuming that dissimilarities are most significant and provided an example of feedback received while implementing projects.

...one thing that I've learned, and literally been taught by people who work with me I've literally have had this said to me: "We're not any different than you. We're human beings." So when people first said you can't take CBT to Africa, the first time I did it, 
people were offended, and were like, 'Why? We think, we behave, we feel. What's the big issue?' I think one of the things that I've learned is that as a Western culture, we try to be so sensitive to cross-cultural variations, and we don't want to force it on anyone. But a lot of the people that I've talked with are offended the other way. 'Why would you think we're so different than you that stuff that works for you as a human being wouldn't work for us?' I've literally heard it every single place I've gone... Even in Africa, they say 'why wouldn't you think this works here? The same drugs work for us. (Participant \#8)

Recognition of similarities and differences also occurs more broadly, such that practitioners are open to differing conceptualizations of health and mental health. Five participants discussed the role of openness as needed to respond to these variations.

What I recognized then was that the kids and families I worked with ended up being very healthy, successful kids and families despite their challenges in the moment. They were very functional, but it just didn't fit my sense of what functional looked like. (Participant \#5)

I've certainly learned a lot about turning off your own conceptualization about mental health and what it looks like and how it's described and how people receive it and how they present. I think I've gotten better at that over time - being open to that. (Participant \#8)

This personal variable of openness, combined with considerations of similarities and differences in individuals and communities, are underlying factors that promote creating responsive programs; in turn, these programs are reflective of specific variations, and yet also appropriately utilize and adapt methods that have provided effective in other contexts.

\section{Collaboration}

Collaboration, with stakeholders, local partners, and service users, was a critical theme that was discussed in eight of nine interviews. Repeatedly, collaboration was identified as a required component for successful GMH projects.

...realize that the only successful outcome here needs to involve collaboration. That to me is the cornerstone. (Participant \#1) 
The main thing I have learned is collaboration. You can't do this work alone." (Participant \#4)

Work as collaborators, not as experts. I think this is probably the most basic. (L. Gerstein, personal communication, March 3, 2017)

Below, subthemes that impact successful collaboration will each be addressed.

Relationship building. Creating relationships emerged as a foundational variable in crafting strong collaborations, with six participants reflecting on aspects of the role of relationships in their interviews. Three participants discussed the importance of relationships as a way of remembering humanity in the midst of GMH projects, as well as the idea of take a longterm view on creating and maintaining relationships.

But build relationships, and see people as humans and don't lose the human side of your work. And this is something that I've heard very powerfully expressed in many global mental health settings... maintaining and emphasizing the relationship aspect of our work. (K. O'Donnell, personal communication, February 13, 2017)

And with these particular projects, there's content that's specific, but one of the most important things is strengthening people to people relationships and cultural understanding. In the long run, you can teach some skills and help develop some infrastructures, but... it's our relationships that are critical. (L. Gerstein, personal communication, March 3, 2017)

It's all about people. It's about developing real relationships and taking a long-term perspective on building relationships. (K. Pike, personal communication, March 10, 2017)

As discussed by one respondent, developing meaningful relationships is also dependent

on one's ability to take the appropriate time and demonstrate patience in designing programs.

You can't go at your American pace where you want something signed yesterday. So I think some of the most successful projects have been the longest because I really took the time to build the relationships, then talk about the project for a long time, really see that we're on the same page, and then go to the next step. Slowly, slowly. (Participant \#2) 
Further, multiple respondents discussed the importance of relationships as a critical factor

that helps to create and sustain interest in projects.

Ultimately, like all of this work, it's stakeholder buy in... I think where relationships were positive and strong, they can sustain the work that it takes to achieve outcomes. Because very often, especially in human behavior change, there's a lot of work and a relatively small change taking place after a long time. So those relationships matter a lot. Where people work well together and they feel excited and confident, and they know they can approach me or anyone in leadership, is good. (Participant \#5)

... such a big part of my relationship is with our supervisors. So in our [country] setting, I was on the phone 2-4 hours every single week for a year and a half with the same person. That's more than I talk to a lot of my friends! And they become really, really close. Even through a 10-day training, I'm there through most of that day, day and night, and you develop very close relationships... I think that creates a lot of buy-in on a very different level. I think that's a real difference there... The longer you are somewhere, the longer you can cultivate those. We do it any, every which way we can. We really do think they're critical. (Participant \#8)

My experience in research and delivery of service outside of the United Sates is that more often than not, you get a real encouraging message from your partners, saying 'oh yeah, we've got to pursue this together. And then it sort of fizzles out after you leave. I think what's different with this one is that we've all invested a lot of energy and time to develop our relationships and maintain them. (L. Gerstein, personal communication, March 3, 2017)

Similarly, building relationships on an organizational level can assist with developing

programs that reflect community needs and can also function to improve sustainability of projects.

Building relationship with the local implementing partner - we spend a lot of time with them. Building capacity, talking about their needs, how long are they gong to be there. Sometimes it's a major NGO, but we try to work with someone who's going to be there forever, so like the local university or a local NGO, someone born and bred in that country. (Participant \#8)

Through these responses, ideas about relationship building focused on the significant power that relationships hold in initiating, creating, and maintaining GMH projects. Of note were 
ideas that suggested that meaningful relationships were those that incorporated a long-term perspective and were responsive to cultural norms and behaviors.

Reciprocity. Creating collaborations that are reciprocal, such that all individuals involved in program development and implementation benefit, was a theme that four participants referenced. For example, one participant discussed shared, shared interest in providing mental health interventions and contrasted this idea against perception about treating mental illness, while also reflecting on the global benefit of improving mental health treatment.

...the idea that mental health can wait because there are other more pressing global health issues is an arrogant and poorly informed view from a privileged high-income country perspective. In my experiences, and I have worked with people around the world, there is significant desire and interest from the lowest income to the highest income countries to improve on understanding mental illness and providing interventions to prevent and treat mental illness." (K. Pike, personal communication, March 10, 2017)

On a program level, this idea of reciprocity emerged within the context of approaching collaborations with the belief that all individuals in partnerships can learn from one another and that each person brings specific knowledge and expertise to programming efforts. Of note, one respondent discussed this concept in terms of finding balance between global knowledge and local expertise.

...my framework for global mental health is that the goal is to bring together global knowledge and local expertise. That's the way to have maximal impact and to insure that people who are working locally and on the ground and have the expertise of the environment and community have access to global knowledge. The global community is informed by local expertise and experience, so I think it really is true that global mental health has to be understood as global network of local experts. (K. Pike, personal communication, March 10, 2017)

Similarly, a different respondent reflected on the intersection of professional knowledge and local expertise, again with the goal of sharing direction of projects among all team members. 
So it's an approach not a 'my knowledge is better' or 'I know more or 'this is the way things are done'. But it's 'this is what I have to bring to the table' and it's something that we're sharing among all of us. (Participant \#5)

Collaborating closely, certainly, but with an eye for being a resource instead of directing. And creating an actual partnership. Because I actually don't know better what they need. I might know specific things about psychology that I can bring to the table, but what they may look like in a small town on the Atlantic coast in Nicaragua is for them to decide, and they can provide that leadership. So I can bring some information, some skills, but it really needs to be about their leaders being asked. (Participant \#5)

Many times in these projects we go prepared, but it's also important to engage our stakeholders. It's not like we're going in with 'here's how we're going to do it.' We're getting all of this input from our stakeholders. (L. Gerstein, personal communication, March 3, 2017)

Further, inherent in some of these statements are also reflections on regarding the role of power and efforts to create collaborations such that power differentials are minimized, with reciprocity identified as a mechanism to move towards equality in partnerships.

And the flip side is an openness from my colleague there about me and appreciating what I have to offer. I think it's a very honest and flat relationship in terms of power. (L. Gerstein, personal communication, March 3, 2017)

We're particularly focused on marginalized groups. And that's another reason to involve them, because it empowers them. It's a good parallel - doing valid and relevant and effective cross-cultural work should be driven by social justice principles, strategies, and philosophies.” (L. Gerstein, personal communication, March 3, 2017)

[Reciprocity is] a bit jargony, but essentially what we're saying is building a respectful, trusting, equal, professional collaboration, where we recognize that both sides have something to contribute and both sides having something to learn, and we're able to do better work together. (K. Pike, personal communication, March 10, 2017)

Paying attention, then, to beliefs about the role of resources and expertise also becomes an area to reflect about perceptions of power and the role of reciprocity to challenge power differentials. 
One of the biggest lessons that I've learned is that being low-resourced doesn't mean lesser, for lack of a better word. And one of the biggest lessons out there is you see people working in really difficult, impossible, challenging environments with virtually no resources, and they do amazing work. They do art therapy, but it's with plants and dirt and things they can find for free. Or there's no actual infrastructure, no buildings, but they find a way to do therapy. They develop community support. So maybe they don't have PhDs, but they're very talented and knowledgeable in the things they do. I think that shows that higher-resourced doesn't mean higher skill or knowledge. And really stopping and paying attention to a culture that is different from our own is valuable. (Participant \#5)

Finally, while there are multiple methods through which to work towards reciprocity (e.g., global and local perspective, equality in relationships), one participant discussed this concept in terms of establishing roles on teams and regarding authorship on resulting publications.

...it's really important for the high-income country to create legitimate partnerships that offer real opportunities for leadership and authorship for low-income countries, and that there are agreements about how to insure that individuals within the partnership are all able to count on whatever it is they - to be able to articulate their needs and be supported in their needs. (K. Pike, personal communication, March 10, 2017)

Communication. The development of meaningful collaborations is directly impacted by regular and ongoing communication. This concept was described by one individual, who stated that "regular communication, given that people are busy and all over the world, having regular communication that keeps people connected to progress and problems is helpful" and can also help to establish a "shared vision" of collaborations (K. Pike, personal communication, March 10, 2017).

Conversely, one participant discussed potential errors that can arise due to inadequate communication, particularly due to varying cultural norms.

And it's tricky - one of the reasons that my initial project in another country fell through was - I thought I was doing everything, crossing my t's and dotting my i's and having 
all these conversations and all. But still, because it's cross-cultural, there's such a potential for misunderstandings or miscommunication. So learning from that, I think there is a hyper-communication that needs to occur when you're working crossculturally, because even if you say "so we're both going to do this"... it doesn't necessarily mean that you're on the same page. So there are different expectations and mindframes. (Participant \#2)

The challenges are that many times because it's outside of your frame of reference, you may not have any idea that something is going to be taken a certain way, or that something you said means a certain thing, or something they said means a certain thing. There's such a meta-cultural context that unless you've been round in that culture, you're just not going to know... a potential for misunderstanding. One way to get around that is to be as genuine and transparent as you can. (Participant \#2)

In addition to the interpersonal variables described above, participants also discussed other recommendations to support meaningful communication and to avoid communication mishaps.

So really, really - don't be in a hurry - take more time rather than less time, even if it's overkill, to ascertain that we all get this, we all understand what we're talking about. (Participant \#2)

I think part of it is the strategy that you use when you're working in international contexts and the way you approach this conversation so that you try to go with the idea and ask a lot of questions and listen more. And when I have an initial reaction that is kind of a 'no', I have to really force myself to stop and think about that, and ask a little more. (Participant \#5)

Finally, one participant reflected on the importance of embracing difficult conversations and developing competence in managing difficult issues that arise.

I think one of the great things about international mental health is that those of us who do this are willing to put ourselves in uncomfortable places. It's great, it's exciting, but it's also uncomfortable. I think if we're willing to be uncomfortable, and we find other people who are willing to be in that space, it also helps to facilitate difficult dialogue. We learn to talk to each other about topics that are uncomfortable because we have been there, and we respect that space. We value the lack of comfort, because we understand it teaches us something, and hopefully our goal is to create a better world. (Participant \#5) 


\section{Program Level Characteristics}

Beyond the two overarching themes described above, respondents also identified multiple variables that operate at the program level. Each will be discussed in more detail below.

\section{Sustainability}

Of note, sustainability was discussed in eight out of nine interviews; in many ways, sustainability appears as a core variable that contributes to successful projects. Multiple participants mentioned the importance of assessing from the outset how to incorporate long-term sustainability plans into their project designs. One participant described this concept in the following way: "From the get go, I'm thinking how [my organization] is going to pull out of this so people don't even know that we're gone. Things are still existing, and things are still running and running well" (Participant \#8). In addition to working at the individual and community levels, some respondents also described the importance of involving actors in higher-level systems, such as governments, to continue advancing the agendas of their projects, again reiterating the importance of building these partnerships from the beginning of their endeavors.

Make sure we make the links between the project and those who will be responsible for the uptake after the project with the big players, policy makers, administrators, and all that. (Participant \#1)

You really need to network with friends and colleagues who are doing this work, and engage with governments, industries and NGOs from very early on, because they are much more likely to carry the work forward when they're part of the process from the very beginning. (K. Pike, personal communication, March 10, 2017)

In addition to linking together communities and individuals to promote sustainable interventions, one participant also reflected upon the importance of creating global networks of interventions to minimize lost resources. In this context, sustainability can be considered both the 
maintenance of existing projects, as well as ongoing ability (i.e., due to available resources) to continue engaging in outreach efforts.

The other takeaway is that we run the risk of expending very precious resources ineffectively if we don't work globally, in terms of engaging a global network, because people are repeating studies that have been done in 30 different places and not using state of the art methodologies, and ultimately not really building a coherent and maximally powerful platform of knowledge. (K. Pike, personal communication, March 10, 2017)

Further, one individual reported that including a sustainability plan has ethical ramifications, particularly so that efforts to improve health in communities are actually creating significant change.

I think actually it's unethical to do work that engages communities where there's not a sustainability plan, because even if the sustainability plan is limited, [you need] to know what are you going to do when this funding is over, and how is that going to be ok, and in fact advance health in the community. (K. Pike, personal communication, March 10, 2017)

Similarly, a different respondent also considered the concept of leaving behind programs that create change and the role that communities play in continuing endeavors.

The idea of the collective is something that has long-term transformative powers. So the idea is that ideally we work with groups and leave them better off than when we started, with resources to continue to drive changes in their own worlds, even after we've left. (R. Burgess, personal communication, March 17, 2017)

While sustainability is critical, projects must have cultural relevance for longevity of programs to be of value to targeted communities. One individual described that "if you're going to start a program, it should be sustainable. It should certainly be flexible and be able to adapt over time. I think we know that programs aren't sustainable if there isn't cultural relevance and reciprocity" (Participant \#5). In response, the concept of involving community members such that projects are valued becomes critical; with this involvement, several participants noted that 
having communities take over leadership of projects is a valued perspective. For example, one participant stated that "the best thing to show how successful this [project] is is to leave something, to make ourselves redundant, so that communities take over and use it in ways that are helpful" (Participant \#1). Another individual described this by stating "in the long run, their voice is really critical to everything you do, and honoring it is going to make things much smoother. It increases the likelihood that once you're not as intimately involved, they're still doing the project" (L. Gerstein, personal communication, March 3, 2017)

\section{Evaluation and Research Methods}

Evaluation and research methods was another frequently mentioned variable, emerging in eight interviews. One participant stated the following: "Evaluate, don't leave things without evaluating them. We have so many surprises in this work - things that felt right ended up being not particularly useful. And we always need to see if they're harmful”' (Participant \#1).

Additionally, the importance of openness was illustrated through statements describing the need "to accept critical feedback or be redirected if you're wrong" (Participant \#2) and be willing to learn from past mistakes (Participant \#8).

Additionally, three participants stressed the importance of ensuring that metrics used in evaluation were both culturally relevant and valid in a given population, noting that revalidation of psychometric tools is an important part of the evaluation process. One individual sated that "your program evaluations should be embedded in your context, not based on US norms or anything of that sort" (Participant \#5).

Further, mechanisms to develop and evaluate programs should also be relevant to local contexts. 
In the United States, valid research is a clinical trial with a huge sample... a lot of people don't conceptualize problems in that way, with problems and hypotheses. So I am much more appreciative of the value of information and data even if they don't look like the kind of information and data that we see in the United States. So sometimes it would feel very fluffy or theoretical for many Americans, but I guess I've learned that it doesn't decrease its value. Maybe it's a unique approach compared to what we're used to, so that we need to give it equal or a greater value. It's made me appreciate science in a different kind of way. (Participant \#5)

There's a US centric ideology that permeates everything that we do in the broader field. If you have that, then you cannot appreciate the way things are done methodologically or conceptually or in terms of intervention elsewhere. So if you go there with 'oh, you have to control all this stuff' or we have to conduct the study this kind of way... my big thing is respecting indigenous culture, respecting indigenous methodology, ways of science, theory, practice. If you're going to do that, then it means you're not going to impose what we've got here. (L. Gerstein, personal communication, March 3, 2017)

Thus, by building evaluations and research projects that are meaningful and respectful to contextual variables, programs offer more value to local communities.

Finally, one participant elaborated to advocate for research methods that empower communities, suggesting that even methodologies that are used in program design have implications for improving mental health.

What makes projects like these successful comes down to the methodologies that you use... a lot of the methods I use are participatory in nature...these tools help to engage communities in unpacking and making tangible their views of the world and their own perspectives, so I use that as a way of engaging and asking people about their lives or types of services they might like... I find this shifts the power a bit so people feel like 'I am doing something, I can do this.' The data collection process itself helps people feel changed by it, empowered by it, to see themselves as producers of knowledge because it's tangible and there and it's not jut a conversation that happens and you never see them again. (R. Burgess, personal communication, March 17, 2017)

\section{Flexibility}

Concepts related to flexibility included managing the unknown, adapting and adjusting approaches, and having an ability to problem solve. One respondent described the need for flexibility 
by saying that "then we're going there thinking it's going to be like this, and then you get there and it's like whoa! You have to make a complete 180, and it has to be seamless, not you moaning and groaning about what's happened" (L. Gerstein, personal communication, March 3, 2017). Further, another individual noted that "you have to change, you have to be able to do a number of things, you have to be able to see a diverse number of problems pop up and go with the flow" (Participant \#8).

In these examples, flexibility is viewed as a skill that allows psychologists to tailor and adapt prior conceptions and plans to realities of the environments where they work. One individual reflected on the reasons that flexibility is needed and the role of openness in mediating flexibility.

So I'm saying, they're saying something, and I'm trying to give some examples. But it just didn't fit into the way that learned to do what they can do. It almost went alongside, but wasn't really crossing over perpendicularly. In that case - I think the other part of being successful - is being really flexible. It's stepping back. It's not plowing through... I need to rethink it, and find a way where we're all speaking the same language. There's another way to talk about this idea. Is there another idea that might have the same outcome? If I was approaching this, my idea would be this, but that doesn't seem to be making much sense. But maybe another approach will, if my ultimate goal is staying focused on that big picture goal. Maybe you can mold your approach to meeting that goal. (Participant \#5)

Finally, outside of interactions with communities, one respondent discussed flexibility as necessary in the early planning stages of projects. Thus, from initial to end stages of projects, flexibility is considered a needed component of successful and useful interventions.

Critical is knowing this project will have 7 lives - it will not get funded the first time, or you'll think you have a partner and that partner will fold, or you have to continue to push forward with the core value and ideas, knowing that it's hard to keep - you have to keep evolving. (K. Pike, personal communication, March 10, 2017)

\section{Attention to Systems}

The importance of designing programs that consider systemic factors that impact mental health, and not only individual level factors, was a global theme from respondents. In particular, 
one author talked about the importance of attending to group dynamics and group behavior,

instead of only targeting interventions to individuals.

But there might be another way to think about psychology, and that is the collective whole. The behaviors and mental processes of collective wholes, of groups... but if we can expand our definition of psychology to include group behavior, and to look at negative life events and poverty, and how poverty is a structural inequality can impact individual and group behavior, then I think psychology has a lot to offer mental health around the world. (Participant \#4)

And the reason I say these interventions are being designed incorrectly is because the movement for GMH does not conceptualize community fully. It thinks of community as a target group as in public health - who is the at risk population, what is the geographical location at which services will be distributed. But it doesn't think of communities as acting, thinking, doing, complex entities where someone might ascribe to a diagnostic category or they might reject it. (Participant \#9)

Beyond group level dynamics, several respondents also spoke of considering higher-level systems (e.g., structural inequality, poverty, sociopolitical context) that affect mental health and deserve consideration in program planning.

We need to continue to develop our special skills, our implementation science, cultural relevance, applying treatments and closing the treatment gap, but it's time to increasingly devote more resources to the underlying causes of mental conditions - things like social determinants of health, health inequalities, poverty. These, I think, are what largely contributing to mental health issues. (K. O'Donnell, personal communication, February $13,2017)$

At its $[\mathrm{GMH}]$ heart, it is about redressing inequality. So certainly our methods and perspective should be one that is interested in a change that is about more than just the individual and is longer than a treatment program. Because if you're only thinking about a treatment, we know that people are not perfect adheres. So if your only interest is getting people to take a treatment, that is insufficient because you've already acknowledged this whole problem exists because of global inequality... and not just the inequality of access and the individual inequality, but the inequality of structures and societies and communities. (R. Burgess, personal communication, March 17, 2017)

Structural violence and geopolitical power and economic structures in societies drive mental health problems, but still we are locked in treating conditions, as if bodies exist in 
isolation from society, as if they are solely biological or psychological bodies. (R. Burgess, personal communication, March 17, 2017)

\section{Multidisciplinary Teams}

In order to meet the complex needs of GMH, and to account for all of the factors that impact mental health, the concept of utilizing multidisciplinary teams was common among respondents.

It matters because we are so under-capacity in terms of reaching the needs, meeting the needs of individuals with mental health concerns around the world. We need to engage all disciplines that are associated with this work... in a way that would provide the most comprehensive understanding of mental illness and most comprehensive planning for addressing the needs. (K. Pike, personal communication, March 10, 2017)

Similarly, a different participant stated that "in order to do global mental health well, we have to be regularly connecting with other sectors - humanitarian development, economic, and so forth” (K. O’Donnell, personal communication, February 13, 2017).

In addition to a larger systems viewpoint, one individual described the importance of a multidisciplinary approach from a project team perspective.

I think teamwork, especially in GMH... I couldn't do any project I do without at least 4 other people. I couldn't write the grant, I couldn't run the budget, I couldn't pull off fieldwork... I think that's one of the big lessons - trusting myself and making sure there's a good team." (Participant \#8)

\section{Clinical Knowledge and Perspective}

Additionally, respondents discussed the importance of needing sound clinical knowledge that informs developing sound treatment platforms.

So with the greater, improved developments in terms of evidence-based treatment - both in terms of psychotherapy and medication treatments - we have treatments that work, and we have a major burden that could be significantly ameliorated with appropriate treatment delivered. (K. Pike, personal communication, March 10, 2017) 
However, one respondent spoke of the interplay between knowledge and personal/team

attributes as an area of competence.

I think we look at best practices internationally, we look at knowledge - we definitely want to be competent in the field and what we do. I think, however, that for a person to be effective in sharing that knowledge, you need an international perspective that incorporates humility and curiosity and an interest in others. (Participant \#5)

Interestingly, while there are a variety of mental health professionals engaging in $\mathrm{GMH}$, one participant discussed the lack of project team members who have explicit attention working with individuals with mental illness on an individual, clinical level, overall advocating for the need of professionals who can use clinical knowledge as a foundation for building relevant treatments.

I'm still surprised how many people do GMH and don't have someone on their team who has actually seen patients. I think for me, for example in my team - when you haven't sat in front of 100 depressed people, you have such a different perspective... I think that perspective from us as clinicians is totally missing still from GMH. They treat it as a public health issue - they're looking at big numbers, massive populations, looking at sustainability. But I think in order to get there, you still need a level of understanding of who are we working with. (Participant \#8)

In sum, successful projects are implemented as team members, with robust clinical knowledge and experience, bring this background into their GMH efforts.

\section{Attention to Spectrum of Mental Health}

Finally, four participants discussed the importance of attending to the whole spectrum of mental health, not only attending to treatment of mental illness. For example, one respondent stated that "instead of focusing on illness and 'craziness', we need to really start thinking about the proactive and positive aspects" (Participant \#5). A different participant expanded upon this theme to suggest that GMH projects "be very committed to well-being - human well-being - not 
just mental health. Be committed to the bigger picture" (K. O'Donnell, personal communication,

February 13, 2017).

Similarly, attention to quality of life was another related factor that emerged. I think most broadly it is a central piece of the work... and when we think about quality of life, there have been a lot of studies that look at symptom remission and not necessarily quality of life. There's a shift toward understanding that you want to implement a treatment that is targeting the core symptoms that are most relevant, but also, even more broadly, if the symptoms aren't going to go into remission or haven't gone into remission, how do we improve quality of life? We improve quality of life by symptom reduction, but even more broadly, how do we improve quality of life and engage in a patient-informed conceptualization of what quality of life means." (K. Pike, personal communication, March 10, 2017)

In part, attention to the spectrum of mental health also creates added focus on prevention, in order to maintain existing health. One respondent indicated that there has been a shift in the field such that there is more focus on "preventing mental health disorders. Not just treating them, but prevention. And explicitly going after that' (K. O'Donnell, personal communication, February 13, 2017).

Critically, attention to the spectrum of mental health does include treatment of and attention to mental illness, as well as maintenance of mental wellbeing. One respondent discussed the importance of finding the interplay of these factors when introducing treatment programs into communities.

So I was thinking should we pay more attention to the determinants of depression instead of trying to deal with the consequences. It became very obvious to me that it's actually critical, not just important, but critical to deal with the mental health of people who are trying to adjust to very difficult conditions, because the resignation that people feel, the constant anxiety, the helpless and hopelessness of anxiety and depression can make people resigned... at a point when they have to be very resourceful for survival - for themselves, for their families. And that's very dangerous." (Participant \#1) 


\section{Individual Level Characteristics}

In addition, many respondents discussed individual level characteristics that impact the value of their GMH work. The skills and practices identified in this section have specific ramifications regarding the quality of work that an individual can contribute both to specific programs and also to moving forward a GMH agenda.

\section{Perseverance}

Need for perseverance was commonly stated by participants, appearing in six of the interviews; this variable related to ongoing GMH efforts despite resistance and challenges of convincing others about the value of the work, difficulties in disseminating work, and a general need to persevere in moving forward a GMH agenda. One individual reflected on past projects and experiences and stated that "I do think you have to be strong enough within yourself to believe in what you're doing, to persevere... it's not the path of least resistance" (Participant \#2). A similar sentiment was echoed by L. Gerstein (personal communication, March 3, 2017), who stated that "there are still some very strong norms not appreciating this kind of work in our field." In light of these roadblocks, one individual discussed the challenges of having to convince others of the usefulness and validity of GMH projects.

With international work, you often have to really convince people of the value of it. So things that you and I would say 'well, of course, they're important!' is not the mainstream view. So you have to be prepared for a bumpy ride. And to realize that your reward is going to often be intrinsic and not extrinsic. (Participant \#2)

Similarly, three participants spoke of challenges in publishing and disseminating their work.

So from the research perspective, editors of journals that act as gatekeepers to crosscultural research that doesn't get published... There are some papers that are extremely well-written, or have a very good story to tell you, but the methodology they used or they 
way they organized it, you know they won't get accepted into a regular APA journal. And yet you know it's a good story that they're telling. (Participant \#4)

And journals reject this stuff all of the time - what research looks like in other places and what that means. Very often journals reject something because the sample was small or something. (Participant \#5)

You don't have the same kind of controls over internal validity. Whenever you do field research, it's much more complex. And I think our journals are not as sensitive to what it takes to do it, and they want to hold us to the same standard as doing research here in a laboratory. It's a big mistake. (L. Gerstein, personal communication, March 3, 2017)

As a result, there is a missed opportunity for the broader community to benefit from GMH-related insights. One individual stated that if “we can't share more through our academic publications, then other people who are not traveling are not only not benefitting, but we're not developing a real emic and epic understanding of human behavior" (L. Gerstein, personal communication, March 3, 2017). Perseverance, then, is needed to face these common obstacles that are expressed above, in order to not allow resistance to diminish one's efforts to move forward in GMH.

Lastly, need to persevere in ongoing efforts and projects was also addressed by one participant, who stated that "change is hard. Creating significant change in a culture, in a healthy system, in a discipline is not easy. Perseverance! It's easy to get discouraged... but you have to look where you've come from. Take a long-term view" (K. O’Donnell, personal communication, February 13, 2017).

\section{Ongoing Mentorship/Supervision}

Of the nine respondents, four identified the importance of ongoing mentorship and supervision as professional competency needed for working within GMH. This most often took the form of having mentors who could provide recommendations and guidance based upon their 
own experiences (Participants \#5 and \#8). In addition, providing support was also valuable, described in the following way: "Encouragement and patience from colleagues who are further along than I am. We need to give each other a leg up" (K. O’Donnell, personal communication, February 13, 2017). Finally, supervision is also an important component of increasing cultural knowledge and competence (Participant \#4).

\section{Self-Awareness}

Developing self-awareness was addressed by four of the participants, particularly in regard to personal motives that might influence GMH projects (Participant \#1), in terms of lack of cultural knowledge (Participant \#1), and about learning to recognize and manage power. Of note was one respondent's statement that there are "many layers of agendas that people have, including ourselves. We need to be very self-reflective and examine ourselves, our motives" (Participant \#1). In regards to power, multiple participants described the need to recognize the power that researchers wield and to manage implications stemming from this power.

And another thing, because of power differentials, people in many other countries are very willing to just accept something from a U.S. researcher, that it's from them so therefore it's right. And you almost have to fight that yourself - you're not only fighting for yourself not to do that, but also for them not to do that. (Participant \#2)

Just because you're a researcher from outside the culture doesn't mean that you're a bad person. It doesn't mean that power structures that you bring are necessarily going to be bad. For example, the notion that a woman should not be beaten every day by her husband might come from the West, but I don't think that's a bad idea... I think it's important that know we're bringing these power differentials in, figure out which ones are more likely to benefit the community and those that are less likely to benefit the community, and act in that way." (Participant \#4)

\section{Boundary Setting}

Finally, the need to set boundaries, both professional and personal, was identified by two participants. In regards to professional boundaries, one individual stated that "professional 
boundaries are important, because you need to be able to let go. And say it is what it is"

(Participant \#4). Just as importantly, Participant \#4 also stated that personal boundaries was equally important, without which "work will chip away at your identity, and all you are is your work. And if your work is draining, where else are you going to go to get replenished?" Another respondent also identified a similar theme related to self-care.

I recommend a sailing image, where you need to take breeze in your sails and move the ship when the wind is blowing, and you're going to need to tack frequently, and you're going to need to ride it out, and be agile and be willing to experiment and be flexible. Know that sometimes you're going to need a lot more energy for personal life or family life, and at another stage in your life you'll have more energy for career pursuits. Ultimately you want to just make sure you have wind in your sails and you're moving forward." (K. Pike, personal communication, March 10, 2017)

Thus, setting boundaries and attending to one's health is critical in that it allows GMH professionals to continue their works and effort and have the energy necessary to continue engaging in their work.

\section{Minor Themes}

Participants identified several minor themes that merit consideration. While these themes were not necessarily recognized by many respondents and thus not guiding principles, the inherent ideas within these responses contain important data to address.

\section{Personal Attributes}

Two respondents ((K. O’Donnell, personal communication, February 13, 2017; and Participant \#5) discussed the idea of personal attributes and the development of character as considerations for psychologists who work in GMH.

One's character is important no matter what you do, and it's part of best practice. I do think we should be emphasizing that, while allowing room for diversity in belief systems and what this means for people. But things like honesty, transparency... are important to cultivate. Courage. Going the extra mile. Sacrifice. All of these things... it's not just 
about professional competence, but also about personal character. (K. O’Donnell, personal communication, February 13, 2017)

Other personal attributes, including openness, curiosity, bravery, and humility, have been discussed previously in this section. However, the idea of developing character as a competency in GMH is intriguing; particularly, how are these attributes developed, and what is the process of character development within the context of GMH best practice recommendations?

Consideration of this idea merits further exploration.

\section{Attention to Complexity}

Attending to complexities of mental health and mental illness was a concept reflected on by two respondents.

Psychologists are able to discuss and understand the neuroscience and sociocultural factors and individual factors and community factors and really come at it from a complex model or capacity for a complex model. (K. Pike, personal communication, March 10, 2017)

So a lot of my approaches to interventions are about moving people away from a primarily biomedical or psychiatric model to what people might call a biosocial model, where you are trying to ensure that your intervention also enables people to have a certain set of competencies that will allow them to achieve well-being in ways that are best for them. And part of that might include being able to access an antidepressant, but it might also be giving guidance on how to get out of poverty or debt. (R. Burgess, personal communication, March 17, 2017)

Particularly, the role of psychologists in considering these variables, instead of only approaching treatments from a biological model, was of emphasis. Developing further insight into how to capture and attend to these complex variables in promoting mental wellbeing and treating mental illness is aligned with recommendations on holistic health, and yet cultivating this perspective in program development is still an area of growth. 
Advocacy

Considering roles of the psychologists outside of developing relationships and projects, specifically in terms of advocating for individuals with mental illness, was discussed by one participant.

Be an advocate, because sometimes people with mental illness in the context of poverty or war or displacement are the expendables sometimes. So it is very important to advocate. (Participant \#1)

This recommendation of advocacy is aligned with existing literature discussing approaches that psychologists might utilize in their work and has possible implications on addressing issues of complexity above (e.g., advocating for structural and systematic issues that impact mental illness). Further, attention to advocacy is a means to advance a social justice agenda, a concept referenced earlier in this section. Examination of how this competency might fit into best practices in GMH, particularly for psychologists, is of interest.

\section{Summary}

Throughout the interview process, it became clear that similar themes were discussed regularly by participants. Generally, common recommendations included focus on broad, overarching variables of projects, as well as on specific program components and individual characteristics. In sum, attention to all levels of these recommendations is posited to result in the most effective, ethical practice of GMH. 


\section{CHAPTER V}

\section{DISCUSSION}

This chapter will review the findings of this exploratory research, address limitations of this study, provide implications of incorporating these findings to address critiques of GMH, and make suggestions for further investigation.

\section{Findings}

This study served to elucidate possible best practice recommendations for psychologists who work within the GMH field, particularly as such recommendations that are targeted to psychologists are lacking. The semi-structured interviews resulted in recommendations on a variety of levels, including overarching variables, program level characteristics, and individual level characteristics.

Notably, the literature review demonstrated that attention to holistic health, cultural relevance, creating partnerships, working collaboratively, and focusing on sustainability are critical aims of GMH program development. Overall, the recommendations stemming from this study are generally aligned with the existing literature, though with a few added considerations and additional focus on the role of the individual practitioner. Further, themes commonly discussed in this study are also aligned with the aims of GMH (e.g., creating partnerships, paying explicit attention to cultural, community, and individual factors that impact mental health). Review of the different categories stemming from this study, as well as support for these recommendations in existing sources, will be reviewed. 
Most broadly and influentially, the overarching variables set comprised recommendations related to consider cultural/contextual variables and collaboration. These variables were viewed as central due to the impacts of these themes on subsequent recommendations. For example, sustainability, which lies within the program level characteristics recommendations, is impacted by both collaborating well with local partners and organizations, as well as by consideration of how to craft programs that have local relevance such that sustainability is valued and useful to individuals and communities. As another example, these impacts are also illustrated by the selfawareness variable within the individual level characteristics recommendations, which suggests the importance of recognizing limits of one's cultural knowledge (i.e., related to development of cultural competence) and recognition of one's power in creating and maintaining relationships (i.e., concepts related to reciprocity and relationship building).

Consideration of cultural and contextual variables, nested within the overarching variables category, was comprised of three subthemes: cultural relevance, cultural competence, and recognizing similarities and differences. As related to cultural relevance, participants in this study identified the importance of responding to community needs, following leadership of local colleagues, and appropriately modifying interventions to fit the context. Developing cultural and contextual competence, evidenced by gaining knowledge of specific environments and responsiveness to norms, values, and beliefs, was also impacted by variables such as respect and humility. Lastly, learning to appreciate and recognize both similarities and differences that individuals and communities express, in addition to varying perspectives of health, was also addressed in this study, and openness was identified as a necessary perspective to meet this aim. These ideas of responding to cultural and contextual concerns was addressed throughout the 
extant literature, with varying degrees of specification and detail (e.g., Boutilier et al., 2011; Khenti et al., 2015; Shah, 2011; Ravitz et al., 2014; Wallcrat et al., 2011).

Collaboration, the other theme within the overarching variables category, included concepts related to relationship building, reciprocity, and communication, and was identified by some participants as the "cornerstone" and "main thing" (Participants \#1 and \#4, respectively). Notably, collaboration should occur not only between project team members, but also between stakeholders, local partners, and service users. Regarding relationship building, taking a long-term view and building into relationships was highly encouraged by respondents. Relationships also function to assist with establishing stakeholder and community buy-in, as well as aid in sustainability. Reciprocity, or crafting interventions and research designs such that all participants involved in interventions benefit, was another concept addressed by respondents. Of note was the concept of finding balance between global knowledge and local expertise; this intersection suggests that while psychologists bring specific professional knowledge of treatments and interventions, effective application of these interventions occurs through direct input and guidance of local community members. Finally, maintaining a strong commitment to communication was identified as a salient aspect of collaboration, with suggestions that patience and ability to tolerate difficult conversations are competencies to develop. Again, these concepts are evident in the existing literature, particularly the role of developing collaborative relationships (e.g., García-Ramírez et al., 2009; Godoy-Ruiz et al., 2016; Khenti et al., 2015), reciprocity (e.g., Godoy-Ruiz et al., 2016; Khenti et al., 2015; Khenti et al., 2012; Raviola et al., 2012), and communication (e.g., Forti, 2005; Kayingo et al., 2016; Larkan, et al., 2016). 
Seven variables from the program levels characteristics set emerged from the data, including sustainability, evaluation and research methods, flexibility, attention to systems, multidisciplinary teams, clinical knowledge and perspective , and attention to spectrum of mental health. These variables each are impacted by the overarching variables described above, and they function mainly at a programmatic level (e.g., impact program planning, impact assessment of project efforts, etc.).

Developing programs with a clear plan for sustainability was a significant construct described by participants. Considering plans for sustainability is necessary from the very beginning of project development, and mechanisms to improve sustainability include involving higher-level actors (e.g., NGOs, governments) and handing over interventions to local communities and individuals. Sustainability serves to decrease ineffective behaviors, such as temporarily "parachuting" into communities and leaving without ensuring long-term benefit, and is so crucial that one participant described ethical ramifications stemming from lack of sustainability planning. Critically, sustainability is only relevant such that programs are meaningful and effective for local communities, linking in recommendations from the cultural and contextual recommendations variables. Likewise, in the extant literature, sustainability is a well-described competency and is also described as needing to be contextually relevant (e.g., Forti, 2005; Raviola et al., 2012; Sapag, Herrera, Trainor, Caldera, \& Khenti, 2013; Shah, 2011; van Ommeren, Saxena, \& Saraceno, 2005). Using relevant evaluation and research methods was another theme stemming from this study, which is also reflected by existing recommendations (Fernando, 2012; Patel, 2014; Sapag et al., 2013; van Ommeren, Saxena, \& Saraceno, 2005). Namely, being culturally and contextually responsive was of note in this category, such that both research design and 
evaluation methods are relevant to local contexts, and application of this recommendation requires openness to variations in how research is conducted. One implication is that via collaboration, utilizing such appropriate methods can serve to empower to communities.

Flexibility, particularly managing the unknown, adapting and adjusting approaches, and learning to problem solve, was commonly discussed by participants. Flexibility serves to assist psychologists in tailoring interventions to communities, instead of rigidly holding on to ineffective approaches, as well as move with and respond to inevitable challenges that arise. The role of flexibility is also discussed by multiple authors (e.g., Fricchione et al., 2012; Godoy-Ruiz et al., 2016; Kayingo et al., 2016), with emphasis on the need for crafting mutable programs that respond to real-time problems and cultural/contextual differences.

Several participants recognized the necessity of attending to systems (i.e., communities and groups, governments, systemic influences of mental health, including poverty and sociopolitical context). In part, this recommendation related to conceptualizing mental health needs outside of the individual and beyond a purely medical lens, such that other drivers of mental health are identified and strengthened. This concept is aligned with GMH's emphasis on addressing inequality (e.g., Patel, 2012); further, this idea is reflected in Khenti et al.'s (2015) recommendation of attending to the holistic health, or context, of individuals.

In order to provide the most comprehensive care, creating projects staffed by multidisciplinary teams was recommended as an important consideration, a recommendation that has been made in existing sources (Boutilier et al., 2011; Raviola et al., 2012; Sapag et al., 2013). Outside of specific projects, it was also suggested that a multidisciplinary approach includes attending to higher-level sectors (e.g., economic) to address all factors related to mental health. 
Need for robust clinical knowledge and perspective emerged as another topic of consideration regarding working in GMH. Psychologists might offer specific expertise and clinical background in this context, as some GMH carries more of a public health focus and has fewer professionals working within it that have specific clinic knowledge. Of note was the idea that working to share this knowledge well is predicated upon individual characteristics such as humility and curiosity. Interestingly, developing projects that are guided by clinical expertise is not a concept described in the existing literature. In part, this might stem from a supposition that GMH projects are foundationally guided by clinical knowledge. Nevertheless, further explicit attention to this construct may be of use.

Finally, attending to the to spectrum of mental health, from mental wellness to mental illness, was described as an important approach to include in GMH efforts. This category included commentary about incorporating prevention efforts and attention to quality of life, as defined by individuals and communities, into outreach designs. While the bulk of the existing literature appears to focus more heavily on treatment of mental illness, there are nevertheless authors who already have advocated for taking a more inclusive approach to treatment (e.g., Campbell \& Burgess, 2012; O’Donnell, 2012; Verdeli, 2016).

Moving to the individual level characteristics recommendations, four recommendations were brought forth by participants, including developing perseverance, ongoing mentorship and supervision, use of self-awareness, and need for boundary setting. Notably, these individual level characteristic are less commonly identified in the existing GMH literature, which tends to focus on broader program or systems-level recommendations. 
Perseverance emerged as a common perspective needed when working in GMH, with implications ranging from maintaining efforts in GMH efforts despite challenges of convincing others about the value of the work, resistance in disseminating work, and difficulties of moving forward a GMH agenda. Perseverance was described as necessary both in terms of career, but also in terms of continuing on with programs despite challenges and setbacks that are frequently encountered along the way. While considered to be vital from participants in this study, perseverance, and most other individual level variables, was not identified in the exiting GMH literature.

Ongoing mentorship and supervision was also identified as critical when working in GMH; this particularly relates to working with psychologists working alongside other professionals who can provide recommendations, guidance, and support, as well as assist with helping psychologists increase their cultural knowledge and competence. While this recommendation relates directly to professional supervision and consultation, this concept is not as clearly defined in the GMH literature. More typically, supervision is discussed regarding psychologists and other mental health professional providing supervision to lay counselors (e.g., Kakuma et al., 2011; Patel, 2012). Nevertheless, need for lifelong learning and ongoing development, particularly in regards to ongoing development of cultural competence, is a concept reflected in other areas of psychological literature (e.g., Heppner, 2006) and deserves attention in GMH.

Developing self-awareness, in regard to personal motives that might influence projects, cultural knowledge, and power, was also identified as important construct in effective GMH efforts. While established in the psychology literature (e.g., Arthur \& Achenbach, 2002; APA, 
2017; Whaley \& Davis, 2007), this construct of attending to self-awareness is not as prevalent or well described in GMH. However, this concept has implications for psychologists attempting to create ethical, culturally relevant projects in ways that are demonstrative of the collaborative approaches described above.

Lastly, learning to set boundaries, both personal and professional, was identified as another recommendation for psychologists. Significantly, setting boundaries and attending to one's health is critical, as it allows GMH professionals to continue their efforts while maintaining their own energy and health. In the GMH literature, discussion of this concept is largely lacking, and yet seems highly relevant to the challenging environments and situations that psychologists often encounter in their work; noticeably, this idea is reflected in the broader psychology literature (e.g., Figley, 2002; Wise, Hersh, \& Gibson, 2012).

Finally, three minor themes (personal attributes, attention to complexity, and advocacy) referenced by participants were of note, though these themes were not universally acknowledged by respondents and thus not main themes. Nevertheless, these concepts have implications to consider and contain important data.

Personal attributes, referenced at various points above, were often described by participants as needed in their work. Examples of these attributes include concepts such as openness, curiosity, bravery, and humility. Attention to these personal qualities, as well as development of character, appears to be understudied in the GMH literature, and yet recognition of these variables was interwoven through many of the comments provided by participants.

Attending to complexity of mental health, from neurobiological and individual level factors to broad systemic factors, was identified as critical in GMH. In many ways, this concept 
is reflected in growing attention to determinants of mental health and illness, as well as clear suggestions about involving higher-level structures (e.g., governments) and attending to variables such as poverty and violence) (e.g., Campbell \& Burgess, 2012; Shah \& Beinecke, 2009). Developing further insight into how to adequately attend to these complex variables in promoting mental wellbeing and treating mental illness remains as an area of further exploration in developing best practice recommendations.

Considerations of advocacy deserve attention, with wide-ranging impacts spanning from increased funding for mental health, attention to the realities of individuals with mental illness, and redress of structural and systemic issues. While only briefly mentioned by participants in this study, GMH literature pays more attention to this construct and recognizes advocacy as mechanism to move towards increasing access to treatment and elimination of health care disparity (e.g., Patel, 2012; Thornicroft et al., 2012). Campbell and Burgess (2012) suggest that increased involvement from community members and development of sound partnerships remain an area of growth for GMH. Outside of GMH literature, advocacy is also described as a means to expand the role of psychologists and work towards meaningful change (Vera \& Speight, 2003). Thus, further exploration of the intersection of GMH, advocacy, and best practice appear to be an area of future growth.

\section{Study Limitations}

Several limitations should be noted before reviewing the implications of this research. First, this qualitative study was designed to produce rich, descriptive results that could begin to formulate recommendations of best practice for psychologists who work within the field of GMH. Nevertheless, the findings from this study cannot be generalized to a broader population. 
However, this study can be used to begin to conceptualize recommendations and codify competencies needed for ethical, effective, and responsive practice of GMH.

Further, the method of recruiting participants (i.e., those identified as experts in the field) was chosen to capture the voices of experienced clinicians and researchers in the GMH field. While respondents were given the opportunity to recommend other peers for inclusion in the study, no additional participants were added to the protocol via this method. As such, it is possible that this study missed including other differing views into the analysis or that all viewpoints from other experts in the field were not included. Efforts to oversample from less well-represented populations (i.e., less reliance on individuals who publish frequently) might have added to the diversity of the sample.

Additionally, all participants included in this study were based in high-income countries, and all communications were conducted in English. In response to ideas of reciprocity and cultural relevance described in this manuscript, learning and hearing the perspectives from psychologists in LMICs is a critical aspect of advancing these recommendations to be more inclusive and reflective of best practice recommendations from a global viewpoint. One mechanism through which to meet this goal could be through the use of translators in order to minimize a possible barrier to participation.

Due to the nature of this qualitative study, all data collected was via self-report from participants. While useful, incorporating other data points to triangulate this information could have served to further confirm findings. For example, this process could have occurred through reviewing documentation from past GMH projects directed by participants or confirmatory interviews with participants or service users impacted by these efforts. 
Finally, while participants were given the opportunity to provide feedback on themes generated from their interviews, only five participants provided approval and/or feedback on the content of the interviews and resulting themes. Thus, while attempts were made to consult and verify interpretations with participants, multiple respondents did not confirm the accuracy of the investigator's interpretations.

\section{Implications of Recommendations}

Findings from this study have a variety of implications that can further promote meaningful practice of GMH. First, while considerations related to cultural and contextual competence are replete in the literature, this study highlights these variables as core components are effective project design, implementation, and evaluation, rather than peripheral issues that are tacked on to projects. Sustained effort and attention to these themes, combined with development of meaningful collaboration, has the potential to address the very critique of GMH that posits its lack of attention to cultural and contextual variables; while some authors have suggested that GMH does not fully address these variables, cultural and contextual competence was identified as critical by all respondents. For example, many participants in this study addressed the need to understand and respond to expressed community needs, rather than needs that outside professionals might assume. In fact, this is one approach recommend as a way to improve upon GMH practice (e.g., Fernando, 2012).

Further, utilizing existing methods of coping, strengthening mental wellbeing as well as mental illness, and attending to systems issues were other concepts identified by respondents, all of which were directly aligned with suggestions in the literature to combat ongoing critqiues. Other concerns, such as those related to power (e.g., Campbell \& Burgess, 2012) might also be 
mitigated via the intersections of consideration of cultural and contextual variables combined with self-awareness, reciprocity, and communication.

Recognition of the drivers of mental health and mental illness, including the interplay of issues that impact health and attention to systems, were also noted as critical in this study. The role of the psychologist in recognizing and attending to these very complexities was identified by respondents and is perhaps a critical role that psychologists can bring to projects. Additionally, robust clinical knowledge and expertise, both in terms of individual and community level factors, was identified as main competency. Together, these perspectives may assist psychologists in bridging the gap between attention to both individual and group level factors.

Lastly, this research underscored variables that lead to successful projects, in large part via attention to culture and context, relationship building, self-awareness, humility, and communication. While some perceive GMH as doing little to adapt practices for local communities (e.g., Kirmayer \& Pedersen, 2014), participants in this study clearly articulated significant efforts to craft programs that are specific and tailored to local community needs. Perhaps part of this disconnect (i.e., concerns about the field versus the reports of practitioners) emerges due to difficulties in publishing and perceived lack of interest in global work, a concern identified by several participants. Moving forward, greater consideration of GMH efforts from the broader psychological community may result in enhanced sharing of efforts and project design to move towards eliminating ongoing concerns.

\section{Suggestions for Further Investigation}

In order to create thorough guidelines for best practice for psychologists in GMH, recommendations for further investigation are as follows. First, recreating this study with a more 
geographically diverse sample (i.e., addition of psychologists from LMICs, including nonEnglish speaking participants) would create a more representative compilation of recommendations. It is quite possible that individuals in different contexts, and with potentially varying educational and professional experiences, might offer considerations that were not articulated in this study. As the goal of these recommendations is to move towards a unified set of recommendations for best practice, including the voices of psychologists from diverse contexts is needed.

Additionally, while these best practice recommendations are specifically designed for psychologists, one area of growth relates to involving community members and service users in the discussion of what constitutes best practice and effective programming. Such an approach is responsive even to some of the themes articulated in this study, including efforts to be culturally and contextually relevant and to craft responsive, reciprocal partnerships. Thus, incorporating both providers, communities, and service users would likely add nuance an dimension to the existing recommendations.

Lastly, future attention to the role of individual level competencies remains an area of exploration for future research, and such exploration would build upon and complement recommendations for higher-order systems. Namely, consideration and investigation of the interplay between individual competencies and program level characteristics is of interest. For example, openness, one such individual level characteristic, has implications for adapting research methods and evaluation to be culturally responsive; however, how is this attribute developed in professionals? Similarly, exploring the role of these individuals factors in terms of effectively driving even overarching variables has potential implications, as some of the 
identified personal variables appear to play a role in developing and utilizing overarching competences (e.g., humility was underscored as critical in developing cultural knowledge and collaboration). Further efforts might also focus on how to develop and shape these individual level competencies.

\section{Conclusion}

This study was an attempt to identify necessary skills and recommendations for meaningful psychological practice in GMH. Participants in this study, all psychologists who practiced within the field of GMH, provided insight into their experiences of effective practice and provided specific details of how these competencies impacted their efforts. While recommendations stemming from this study are broadly representative of existing recommendations for mental health professionals at large (e.g., psychiatrists, public health officials), establishing guidelines for effective practice remains an important area of exploration for relevant and ethical practice of psychology within GHM. 


\section{APPENDIX A}

INTRODUCTORY LETTER 
Hello,

My name is Kimberly Hook, and I am a PhD candidate in the Counseling Psychology program at Loyola University Chicago. I am conducting a study on best practices in global mental health for my dissertation. My intention is to gather real-life narratives from psychologists who engage in such efforts, in order to better understand the successes, challenges, and recommendations that come from experience. You are being contacted as you have been identified as an expert in this domain through your scholarship, publications, and/or service work. I would like to conduct one interview (via Skype or phone) that should last between one- to two-hours. The interview will be audio recorded and transcribed for analysis. No identifying information of the interviewee will be included in the audio recording or report. The exception to this is if you desire to have part or all of your interview attributed to you, due to your expertise in the field; however, this decision is solely up to you. The interview will take place at a mutually agreed upon time.

If you are interested in this study, please email Kimberly Hook at khook@luc.edu. I will follow up with you regarding next steps. In case you would like to review the interview questions before making a decision on study participation, please let me know. If you have further questions or concerns, please feel free to contact me or my advisor (Dr. Elizabeth Vera; evera@luc.edu).

Your participation would be greatly appreciated.

Sincerely,

Kimberly Hook, MA 
APPENDIX B

CONSENT TO PARTICIPATE IN RESEARCH 
Project Title: Best Practices in the Practice of Global Mental Health: An Exploratory Study Principal Investigator: Kimberly Hook, MA

Faculty Sponsor: Elizabeth Vera, $\mathrm{PhD}$

You are being asked to take part in a research study being conducted by Kimberly Hook for a dissertation under the supervision of Dr. Elizabeth Vera in the Counseling Psychology Program at Loyola University of Chicago. You are being asked to participate because of your expertise in the field of global mental health, as evidenced by your scholarship, publications, and/or service work. You are one of approximately 12-20 participants being interviewed for this study. Please read this form carefully and ask any questions you may have before deciding whether to participate in the study.

\section{Purpose:}

The purpose of this study is to learn about the experiences of psychologists who practice in the global mental health field. Specifically, I am interested in learning about the types of projects that you have worked on, lessons learned through your work (including successes and challenges), and recommendations that you have for psychologists engaging in global mental health practice. This research may provide a better understanding and more effective practices for future global mental health practice.

\section{Procedures:}

If you agree to be in the study, you will be asked to participate in a one- to two-hour interview (phone or Skype) regarding your experiences as a psychologist engaged in global mental health work. The interviewer will ask open-ended questions, providing you with the opportunity to tell your story and describe your thoughts and experiences. The interview will be audiotaped and transcribed for the purpose of analysis and report. You will also be asked to complete a brief demographics questionnaire. Additionally, you will be asked to consider fellow qualified peers who might be able to provide further information about the practice of global mental health for this study; you will be provided with the contact information of the primary investigator (PI), as well as study recruitment materials, and will be asked to pass along this information to these peers so that they may contact the PI if they desire to participate. Finally, after data analysis is completed, you will have the opportunity to review, approve, and/or modify themes from your interview.

By signing below, I indicate that I agree to have my interview audiotaped.

Signature (participant):

Date: 
Risks/Benefits:

There are no foreseeable risks involved in participating in this research beyond those experienced in everyday life.

You are not required to share any information that you are not comfortable reporting. There will be no penalty should you decide to withdraw at any time.

There are no direct benefits to you from participation, but the results of this study will be used to provide recommendations for the practice of global mental health. This research may provide insight into effective practice and research within this domain.

\section{Confidentiality:}

We will not be asking your name on the demographic form and the interviewer will not state your name throughout the course of the interview. Your name or identifying information will be removed from the transcription or report. All demographic information in the final paper will be reported in aggregate. Only the listed researchers and a professional transcriber will have access to the audio files. The audio files will be stored behind a locked door and will be destroyed 6 months after the completion of transcription. The consent form with your signature will be kept separate from the demographic form and audio file. However, due to your status as an expert and if you so choose, you may opt to be identified with all or part of the quotes from your interview. This decision will be left strictly up to your discretion.

\section{Voluntary Participation:}

Participation in this study is voluntary. If you do not want to be in this study, you do not have to participate. Even if you initially decide to participate, you may stop the interview at any time, withdraw from the study, and decline to answer any questions without penalty.

\section{Contact and Questions:}

If you have questions about this research project or interview, feel free to contact Kimberly Hook at khook@luc.edu or the faculty sponsor Dr. Elizabeth Vera atevera@luc.edu. If you have any questions about your rights as a research participant, you may contact Loyola's Office of Research Services at 773.508.2689. 
Statement of Consent:

Your signature below indicates that you have read the information provided above, have had an opportunity to ask questions, and agree to participate in this study. You will be given a copy of this form to keep for your records. Please return this form to Kimberly Hook (khook@luc.edu) via email.

Participant's (Your) Signature

Date

Researcher's Signature

Date 


\section{APPENDIX C}

CONSENT TO PARTICIPATE IN RESEARCH: INTERVIEW ATTRIBUTION 
Project Title: Best Practices in the Practice of Global Mental Health: An Exploratory Study Principal Investigator: Kimberly Hook, MA

Faculty Sponsor: Elizabeth Vera, $\mathrm{PhD}$

\section{Procedures:}

After your participation in this study, you may choose to be identified with all or parts of your interview in the final study report. This is strictly optional and left to the discretion of the study participant.

Please mark which applies:

I want my whole interview attributed to me.

I want the selected parts of my interview, as listed below, identified with me.

Selected quotes:

\section{Contact and Questions:}

If you have questions about this research project or interview, feel free to contact Kimberly Hook atkhook@luc.edu or the faculty sponsor Dr. Elizabeth Vera atevera@luc.edu. If you have any questions about your rights as a research participant, you may contact Loyola's Office of Research Services at 773.508.2689.

\section{Statement of Consent:}

Your signature below indicates that you have read the information provided above, have had an opportunity to ask questions, and agree to have all or select quotes from your interview (as indicated above) attributed to you in the final report. You will be given a copy of this form to keep for your records. Please return this form to Kimberly Hook (khook@luc.edu) via email.

Participant's (Your) Signature

Researcher's Signature

\section{Date}

Date 


\section{APPENDIX D}

SEMI-STRUCTURED INTERVIEW QUESTIONS 
1. What has your experience been in the practice of global mental health?

a. What projects have you worked on?

b. Where have you worked?

c. How did you get interested in your work?

2. What do you wish you had known when beginning your career in global mental health?

3. As you reflect on your past work, what do you think are your most important "lessons learned"?

4. What has made the projects or interventions that you have worked on successful?

a. How have you defined "successful"?

5. Tell me about any specific failures or unexpected challenges you have encountered in global mental health work and what you have learned from these experiences.

6. There are some challenges to the practice of global mental health, particularly a.) concerns that it does not truly account for cultural context when attempting to provide interventions and b.) perpetuates issues such as power differentials and lack of involvement of the local community when outside professionals attempt to partner with local communities. How would you respond to these challenges?

7. What would you consider to be essential components of "best practice" when it comes to designing and implementing successful projects?

8. Some recommendations from the existing literature have suggested the importance of considering issues such as "reciprocity, sustainability, holistic health, cultural competence, and improvement of quality of life" in the practice of global mental health.

a. How would you respond to this statement?

b. Have these factors been important to you? Have this factors not been important in your work?

c. What would you add or remove from this list?

9. Are there unique ways that psychology contributes to the field of global mental health to address issues such as mental health disparities? 


\section{APPENDIX E}

DEMOGRAPHICS QUESTIONNAIRE 
1. Age:

2. Gender:

3. Education: $\mathrm{PhD}$ in Psychology. Year of Completion:

Additional trainings or certification:

4. Country of Origin:

5. Racial Identification (select all that apply):

White (Caucasian, European American)

Black (African American)

Asian or Pacific Islander

Hispanic (Latino/a)

Native American

Other (please specify)

6. Current Employer (type; e.g., university, government institution):

7. Funding Source:

8. Number of Years of Field Experience:

9. Type of Global Mental Health work (please provide a brief description of your roles and contexts in which you participate/have participated, including research, clinical, and/or field experience work): 


\section{REFERENCES}

American Psychological Association. (2009). American Psychological Association Strategic Plan. Retrieved from http://www.apa.org/about/apa/strategic-plan/

American Psychological Association. (2012). 2012 CIRP strategic plan and goals. Retrieved from http://www.apa.org/international/governance/cirp/strategic-plan.aspx

American Psychological Association. (2017). Multicultural guidelines: An ecological approach to context, identity, and intersectionality. Retrieved from:

http://www.apa.org/about/policy/multicultural-guidelines.pdf

Arthur, N., \& Achenbach, K. (2002). Developing multicultural counseling competencies through experiential learning. Counselor Education and Supervision, 42(1), 2-14. http://dx.doi.org/10.1002/j.1556-6978.2002.tb01299.x

Becker, A. \& Kleinman, A. (2012). An agenda for closing resource gaps in global mental health: Innovation, capacity building, and partnerships. Harvard Review of Psychiatry, 20(1), 35. http://dx.doi.org/10.3109/10673229.2012.

Berry, J. (2013). Achieving a global psychology. Canadian Psychology/Psychologie Canadienne, 54(1), 55-61. http://dx.doi.org/10.1037/a0031246

Bolton, P., Bass, J., Neugebauer, R., Verdeli, H., Clougherty, K., \& Wickramaratne, P... Weissman M. (2003). Group interpersonal psychotherapy for depression in rural Uganda. Journal of the American Medical Association, 289(23), 3117. http://dx.doi.org/10.1001/jama.289.23.3117

Boutilier, Z., Daibes, I., \& Di Ruggiero, E. (2011). Global health research case studies: Lessons from partnerships addressing health inequities. BMC International Health and Human Rights, 11(Supplement 2), S2.

Bullock, M. (2014). Internationalization in psychology and in APA: A process, not an outcome. Psychology International, 25(1), 6-7.

Bullock, M. (2016). Processes of internationalization. Psychology International, 27(2), 8-9. 
Burgess, R. \& Campbell, C. (2014). Contextualising women's mental distress and coping strategies in the time of AIDS: A rural South African case study. Transcultural Psychiatry, 51(6), 875-903. http://dx.doi.org/10.1177/1363461514526925

Campbell, C. \& Burgess, R. (2012). The role of communities in advancing the goals of the Movement for Global Mental Health. Transcultural Psychiatry, 49(3-4), 379-395. http://dx.doi.org/10.1177/1363461512454643

Canadian Coalition for Global Mental Health. (2015). CCGHR principles for global health research. Retrieved from http://www.ccghr.ca/wp-content/uploads/2015/10/CCGHRPrinciples-for-GHR-FINAL.pdf

Castro, F., Barrera, M., \& Holleran Steiker, L. (2010). Issues and challenges in the design of culturally adapted evidence-based interventions. Annual Review of Clinical Psychology, 6(1), 213-239.

Collins, P., Patel, V., Joestl, S., March, D., Insel, T.R., \& Daar, A.S. (2011). Grand challenges in global mental health. Nature, 475(7354), 27-30.

Commission for Research Partnerships with Developing Countries. (2014). A guide for transboundary research partnerships: 11 principles (2nd ed.). Retrieved from https://naturalsciences.ch/uuid/564b67b9-c39d-5184-9a94e0b129244761?r=20170706115333_1499301166_3898d31d-7a25-55d7-8208d9cbeadald05

Corbin, J. \& Strauss, A. (1990). Grounded theory research: Procedures, canons, and evaluative criteria. Qualitative Sociology, 13(1), 3-21. http://dx.doi.org/10.1007/bf00988593

Creswell, J., Hanson, W., Clark Plano, V., \& Morales, A. (2007). Qualitative research designs: Selection and implementation. The Counseling Psychologist, 35(2), 236-264. http://dx.doi.org/10.1177/0011000006287390

Fernando, G. (2012). The roads less traveled: Mapping some pathways on the global mental health research roadmap. Transcultural Psychiatry, 49(3-4), 396-417. http://dx.doi.org/10.1177/1363461512447137

Figley, C. (2002). Compassion fatigue: Psychotherapists' chronic lack of self care. Journal of Clinical Psychology, 58(11), 1433-1441. http://dx.doi.org/10.1002/jclp.10090

Forrest, L. (2009). Linking international psychology, professional competence, and leadership: Counseling psychologists as learning partners. The Counseling Psychologist, 38(1), 96120 . 
Forti, S. (2005). Building a partnership for research in global health. Retrieved from http://www.ccghr.ca/wpcontent/uploads/2013/05/AnalyticalFramework_BuildingPartners hips_en.pdf

Fricchione, G., Borba, C., Alem, A., Shibre, T., Carney, J., \& Henderson, D. (2012). Capacity building in global mental health: Professional training. Harvard Review of Psychiatry, $20(1), 47-57$.

García-Ramírez, M., Paloma, V., Suarez-Balcazar, Y., \& Balcazar, F. (2009). Building international collaborative capacity: Contributions of community psychologists to a European network. American Journal of Community Psychology, 44(1-2), 116-122. http://dx.doi.org/10.1007/s10464-009-9247-7

Glaser, B. G., \& Holton, J. (2007). Remodeling grounded theory. Historical Social Research, 19, 47-68 .

Godoy-Ruiz, P., Cole, D. C., Lenters, L., \& McKenzie, K. (2016). Developing collaborative approaches to international research: Perspectives of new global health researchers. Global Public Health, 11(3), 253-275.

Heppner, P. (2006). The benefits and challenges of becoming cross-culturally competent counseling psychologists. The Counseling Psychologist, 34(1), 147-172. http://dx.doi.org/10.1177/0011000005282832

Kakuma, R., Minas, H., van Ginneken, N., Dal Poz, M., Desiraju, K., \& Morris, J... Scheffler, R. (2011). Human resources for mental health care: Current situation and strategies for action. The Lancet, 378(9803), 1654-1663.

Kayingo, G., Cobb, N., Meckel, M., Cuadrado, H., Mulitalo, K., \& Fahringer, D. (2016). Collaborating across borders in global health. The Journal of Physician Assistant Education, 27(2), 95-97.

Khenti, A., Fréel, S., Trainor, R., Mohamoud, S., Diaz, P., \& Suh, E... Sapag, J. (2015). Developing a holistic policy and intervention framework for global mental health. Health Policy and Planning, 31(1), 37-45. http://dx.doi.org/10.1093/heapol/czv016

Khenti, A., Sapag, J., Trainor, R., Candia, X., Poblete, F., Valdés, A... Chacon, S. (2012). Strengthening efforts to integrate mental health into primary health care in Chile. International Journal Of Mental Health, 41(1), 87-102. http://dx.doi.org/10.2753/imh0020-7411410107 
Kidd, S., Madan, A., Rallabandi, S., Cole, D., Muskat, E., Raja, S... McKenzie, K. (2016). A multiple case study of mental health interventions in middle income countries: Considering the science of delivery. PLOS ONE, 11(3), e0152083.

Kirmayer, L. \& Pedersen, D. (2014). Toward a new architecture for global mental health. Transcultural Psychiatry, 51(6), 759-776. http://dx.doi.org/10.1177/1363461514557202

Koplan, J., Bond, T., Merson, M., Reddy, K., Rodriguez, M., Sewankambo, N., \&Wasserheit, J. (2009). Towards a common definition of global health. The Lancet, 373(9679), 19931995.

Larkan, F., Uduma, O., Lawal, S., \& van Bavel, B. (2016). Developing a framework for successful research partnerships in global health. Globalization And Health, 12(17).

Lypen, K., Lockwood, N.M., Shalabi, F., Harper, G.W., \& Ngugi, E. (2015). "When we are together I feel at home." Types and sources of social support among youth newly diagnosed with HIV in urban Kenya: Implications for intervention. African Journal of AIDS Research, 14(3), 275-284.

Massachusetts General Hospital Division of Global Psychiatry. (n.d.). Key Characteristics. Retrieved from http://www.mghglobalpsychiatry.org/mission.php

Merriam, S. B. (2009). Qualitative research: A guide to design and implementation. San Francisco, CA: Jossey-Bass.

Meyers, L. (2006). Redemption over revenge: Two psychologist-led programs offer help to Rwanda's walking wounded. Monitor on Psychology, 37(4), 40.

Morrow, S. (2005). Quality and trustworthiness in qualitative research in counseling psychology. Journal of Counseling Psychology, 52(2), 250-260. http://dx.doi.org/10.1037/00220167.52.2.250

Morrow, S. (2007). Qualitative research in counseling psychology: Conceptual foundations. The Counseling Psychologist, 35(2), 209-235.

Murray, L., Dorsey, S., Bolton, P., Jordans, M., Rahman, A., Bass, J., \& Verdeli, H. (2011). Building capacity in mental health interventions in low resource countries: an apprenticeship model for training local providers. International Journal of Mental Health Systems, 5(30). http://dx.doi.org/10.1186/1752-4458-5-30

O’Donnell, K. (2012). Global mental health: A resource primer for exploring the domain. International Perspectives in Psychology: Research, Practice, Consultation, 1(3), 191205. 
Patel, V. \& Prince, M. (2010). Global mental health: A new global health field comes of age. Journal of the American Medical Association, 303(19), 1976-1977. http://dx.doi.org/10.1001/jama.2010.616

Patel, V., Weiss, H., Chowdhary, N., Naik, S., Pednekar, S., \& Chatterjee, S... Kirkwood B. (2010). Effectiveness of an intervention led by lay health counsellors for depressive and anxiety disorders in primary care in Goa, India (MANAS): A cluster randomised controlled trial. The Lancet, 376(9758), 2086-2095. http://dx.doi.org/10.1016/s01406736(10)61508-5

Patel, V. (2012). Global mental health: From science to action. Harvard Review of Psychiatry, $20(1), 6-12$.

Patel, V. (2014). Why mental health matters to global health. Transcultural Psychiatry, 51(6), 777-789. http://dx.doi.org/10.1177/1363461514524473

Ravitz, P., Wondimagegn, D., Pain, C., Araya, M., Alem, A., Baheretibeb, Y...Leszcz, M. (2014). Psychotherapy knowledge translation and interpersonal psychotherapy: Using best-education practices to transform mental health care in Canada and Ethiopia. American Journal of Psychotherapy, 68(4), 463-488.

Raviola, G., Eustache, E., Oswald, C., \& Belkin, G. (2012). Mental health response in Haiti in the aftermath of the 2010 earthquake: A case study for building long-term solutions. Harvard Review of Psychiatry, 20(1), 68-77.

Rosso Buckton, A. (2015). Conversations between anthropology and psychiatry: Drawing out the best from interdisciplinarity in global mental health. Australasian Psychiatry, 23(6 Supplement), 3-5.

Sapag, J., Herrera, A., Trainor, R., Caldera, T., \& Khenti, A. (2013). Global mental health: Transformative capacity building in Nicaragua. Global Health Action, 6.

Saraceno, B., Ommeren, M., Batniji, R., Cohen, A., Gureje, O.; Mahoney, J... Underhill, C. (2007). Barriers to improvement of mental health services in low-income and middleincome countries. The Lancet, 370(9590), 1164-1174.

Shah, S. (2011). Ethical standards for transnational mental health and psychosocial support (MHPSS): Do no harm, preventing cross-cultural errors and inviting pushback. Clinical Social Work Journal, 40(4), 438-449.

Shah, A. \& Beinecke, R. (2009). Global mental health needs, services, barriers, and challenges. International Journal of Mental Health, 38(1), 14-29. 
Strauss, A., \& Corbin, J. (1994). Grounded theory methodology: An overview. In N. Denzin \& Y. Lincoln (Eds.), Handbook of Qualitative Research (pp. 273-284). Thousand Oaks, CA: Sage.

Summerfield, D. (2008). How scientifically valid is the knowledge base of global mental health? BMJ, 336(7651), 992-994. http://dx.doi.org/10.1136/bmj.39513.441030.ad

Suzuki, L., Ahluwalia, M., Arora, A., \& Mattis, J. (2007). The pond you fish in determines the fish you catch: Exploring strategies for qualitative data collection. The Counseling Psychologist, 35(2), 295-327. http://dx.doi.org/10.1177/0011000006290983

Swiss Commission for Research Partnerships with Developing Countries. (2014). A guide for transboundary research partnerships: 11 principles. Retrieved from https://naturalsciences.ch/uuid/564b67b9-c39d-5184-9a94e0b129244761?r=20170706115333_1499301166_3898d31d-7a25-55d7-8208d9cbeada1d05

Thornicroft, G., Cooper, S., Van Bortel, T., Kakuma, R., \& Lund, C. (2012). Capacity building in global mental health research. Harvard Review of Psychiatry, 20(1), 13-24. http://dx.doi.org/10.3109/10673229.2012.649117

Tribe, R., Weerasinghe, D., \& Parameswaran, S. (2014). Increasing mental health capacity in a post-conflict country through effective professional volunteer partnerships: A series of case studies with government agencies, local NGOs and the diaspora community. International Review of Psychiatry, 26(5), 558-565.

van Ommeren, M., Saxena, S., \& Saraceno, B. (2005). Mental and social health during and after acute emergencies: Emerging consensus? Bulletin of the World Health Organization, 83(1), 71-76.

Vera, E., \& Speight, S. (2003). Multicultural competence, social justice, and counseling psychology: Expanding our roles. The Counseling Psychologist, 31(3), 253-272. http://dx.doi.org/10.1177/0011000003031003001

Verdeli, H. (2016). Global mental health: An introduction. Journal of Clinical Psychology: In Session, 72(8), 761-765.

Wallcrat, J., Amering, M., Freidin, J., Davar, Froggatt, D., Jafri, H.,...Herrman, H. (2011). Partnerships for better mental health worldwide: WPA recommendations on best practices in working with service users and family carers. World Psychiatry, 10(3), 229236. 
Whaley, A., \& Davis, K. (2007). Cultural competence and evidence-based practice in mental health services: A complementary perspective. American Psychologist, 62(6), 563-574. http://dx.doi.org/10.1037/0003-066x.62.6.563

White, R., Jain, S., \& Giurgi-Oncu, C. (2014). Counterflows for mental well-being: What highincome countries can learn from low and middle-income countries. International Review of Psychiatry, 26(5), 602-606.

Wise, E., Hersh, M., \& Gibson, C. (2012). Ethics, self-care and well-being for psychologists: Reenvisioning the stress-distress continuum. Professional Psychology: Research and Practice, 43(5), 487-494. http://dx.doi.org/10.1037/a0029446

World Health Organization. (2001). The world health report 2001: Mental health: new understanding, new hope. Geneva: World Health Organization.

World Health Organization. (2011). Mental health atlas 2011. Geneva: World Health Organization.

World Health Organization (2017). Depression and other common mental disorders global health estimates. Geneva: World Health Organization.

WHO World Mental Health Survey Consortium. (2004). Prevalence, severity, and unmet need for treatment of mental disorders in the World Health Organization world mental health surveys. Journal of the American Medical Association, 291(21), 2581. 
VITA

Kimberly Michelle Hook received a Bachelor of Science degree in Health Education and Behavior from the University of Florida in 2009, where she graduated summa cum laude; in 2013, she received a Master of Arts degree in Clinical Psychology from Wheaton College. In addition to completing a variety of clinical trainings and an APA-accredited doctoral internship, she also taught graduate-level courses in psychology at both Loyola University Chicago and North Park University. Throughout her graduate training, she researched topics such as task shifting in low and middle-income countries, social-emotional impacts on English language learners, and impacts of parental monitoring on adolescent substance use initiation. In the future, she plans to focus her professional career on developing and adapting global mental health interventions in under-resourced populations. 